\title{
Spectroscopie neutronique : un outil idéal pour le scientifique des matériaux
}

\section{H. Schober}

Institut Laue Langevin, 38042 Grenoble cedex, France

\section{I- INTRODUCTION}

\section{II- VIBRATIONS DANS LES SOLIDES}

\author{
2-1. Approximation adiabatique \\ 2-2. Modes propres harmoniques \\ 2-3. déplacements quadratiques moyens \\ 2-4. Les phonons dans les systèmes avec invariance de translation
}

\section{III- LES INFORMATIONS VIBRATIONNELLES TESTEES PAR LES NEUTRONS}

3-1. Facteur de structure dynamique

3-2. Densité d'états vibrationnels

3-3. Intensité de la diffusion inélastique

\section{IV- EXPERIENCES DE DIFFUSION INELASTIQUES DES NEUTRONS \\ V- EXEMPLE : SPECTRE DES MODES EXTERNES DES FULLERENES \\ VI- REFERENCES}

Ce article est une discussion concise et auto-consistante de la diffusion inélastique des neutrons appliquée à l'examen des vibrations dans les solides. Les sections d'introduction théoriques sont illustrées par des exemples pris parmi les recherches récentes faites par l'auteur sur les fullérènes.

\section{Introduction}

Dans cet article nous souhaitons discuter comment la diffusion inélastique des neutrons (INS) peut apporter un soutien important à la science des matériaux ${ }^{1}$. Comme il est impossible de couvrir l'ensemble des phénomènes inélastiques, nous nous concentrerons sur l'étude des propriétés vibrationnelles des matériaux non magnétiques.

\footnotetext{
1 Le terme science des matériaux n'est pas d'un usage accepté de façon unanime. Nous définirons la science des matériaux comme toute activité scientifique qui a pour but principal la compréhension des propriétés d'un matériau étudié de manière spécifique et non l'établissement de relations ou de lois fondamentales
} 
Pourquoi un scientifique des matériaux peut-il s'intéresser aux propriétés vibrationnelles? La réponse à cette question est multiple et touche pratiquement toutes les étapes de la recherche de nouveaux matériaux. L'information la plus fondamentale à laquelle on est confronté avec un nouveau matériau c'est certainement sa structure. De ce point de vue la diffraction des rayons X et des neutrons donne l'impulsion fondamentale. Cependant, il existe de nombreux exemples où la connaissance des propriétés vibrationnelles facilite, sinon même est parfois indispensable, pour affiner la structure. Cette affirmation est particulièrement vraie dans le cas de matériaux complexes ou fortement désordonnés où l'affinement jusqu'à des positions atomiques est au mieux très fastidieux et souvent absolument impossible. Une fois connue la structure, les liaisons chimiques peuvent se déduire à partir de considérations sur la longueur des liaisons. Une manière plus directe d'obtenir les longueurs de liaison consiste à déterminer les forces de rappel à partir de l'analyse du spectre de vibration. A partir du spectre vibrationnel on peut dans l'étape suivante calculer des propriétés thermodynamiques telles que la chaleur spécifique ou l'entropie de vibration. Finalement, comme les vibrations testent le système électronique elles permettent de contrôler des changements qui s'y produisent. Par suite, l'INS donne des informations importantes concernant des phénomènes tels que les transitions métal-isolant ou la supraconductivité.

De quelles informations avons-nous besoin et comment les obtenir? Pour un matériau monocristallin, il est en principe possible de retrouver l'information complète concernant les excitations vibrationnelles, i.e. à la fois les fréquences des phonons et les vecteurs propres. Cependant ceci est une tâche fastidieuse et n'a seulement été réalisé que pour des branches de phonons sélectionnées et dans des systèmes particuliers [1]. En conséquence cartographier les relations de dispersion dans des milieux complexes n'est pas une tâche triviale [2,3,4]. Aussi, même lorsqu'on dispose de monocristaux de taille convenable la détermination des relations de dispersion ne peut pas devenir, dans le futur proche, un processus standard pour la science des matériaux. Heureusement dans la plupart des cas on n'a pas besoin d'une information complète sur les propriétés vibrationnelles. Par ex., des propriétés thermo-dynamiques telles que la chaleur spécifique sont définies en termes de densités d'états $G(\omega)$, qui peut être déterminé directement à partir de $S(\vec{Q}, \omega)$ la fonction de diffusion moyennée pour une poudre. Les anharmonicités [5], les changements de phase [6] et les couplages électron-phonon [7], tout cela laisse des traces dans $S(\vec{Q}, \omega)$. Quelques unes de ces empreintes sont suffisamment spécifiques pour s'interpréter sans l'aide de calculs. D'autres nécessitent des modèles grossiers tandis que beaucoup peuvent seulement se déchiffrer avec l'aide des calculs qui sont basés soit sur la mécanique moléculaire, soit sur la théorie de la fontionnelle de densité, soit sur la combinaison des deux. Naturellement le scientifique préfère utiliser des caractéristiques hautement spécifiques. Cependant, grâce aux progrès dans les algorithmes de calcul $[8,9]$ et de leur facilité d'utilisation, la fonction totale $S(\vec{Q}, \omega)$ contient de plus en plus d'informations récupérables. De toute façon, dans le cas de matériaux qu'on ne peut faire croître en monocristaux ou qui sont intrinsèquement désordonnés, $S(\vec{Q}, \omega)$ est tout ce qu'on peut obtenir. Beaucoup de matériaux modernes appartiennent à cette classe.

Pourquoi devons nous utiliser les neutrons pour étudier les vibrations? C'est une question importante car la spectroscopie de diffusion inélastique des neutrons n'est possible qu'auprès des grands instruments et qu'elle est en compétition avec nombre de techniques de laboratoire très performantes, principalement la spectroscopie optique. La réponse réside dans le fait que ce sont les neutrons (et dans une proportion grandissante les rayons $\mathrm{X}$ ) qui sont capables de faire la carte complète du spectre de vibration. Les spectres Raman et infra-rouge peuvent offrir une meilleure résolution en haute fréquence (i.e. dans la gamme des $\mathrm{THz}$ ) mais ils sont limités à la région de $\mathrm{Q}$ autour du centre de zone (i.e. à de très petits transferts de moment) et sont sujet à de très sévères 
règles de sélection ${ }^{2}$. Par suite ils peuvent donner des informations très précises et hautement significatives sur un nombre limité de modes, tandis que les neutrons couvrent le spectre complet, fournissant la densité d'états, les relations de dispersion et même dans certains cas déterminent le schéma des déplacements vibrationnels. Un autre énorme avantage des neutrons sur la spectroscopie optique est l'observation simultanée des changements dans la structure (via le canal élastique), des relaxations (via les signaux quasi élastiques) et des vibrations. Ceci est particulièrement intéressant quand on étudie des réactions chimiques ou des transitions de phase. L'interaction des neutrons avec la matière est très bien comprise. Par suite, il est possible d'interpréter quantitativement les phénomènes de diffusion. Le couplage de la lumière avec les excitations dans un solide est encore un sujet de débat. Dans le cas de la diffusion Raman la situation est encore plus compliquée du fait que les polarisibilités entrent dans l'analyse quantitative. Par suite, l'analyse est souvent réduite à la recherche de la position des maxima. Contrairement à la spectroscopie optique les neutrons ont une très grande profondeur de pénétration et par suite, ils testent les propriétés du cœur du matériau. Ceci est un avantage quand la dégradation chimique - par exemple en fonction de la température- a lieu dans des couches proches de la surface. Ceci a pour conséquence que la diffusion inélastique des neutrons exige de grands échantillons. Nous ne voulons pas poursuivre par une comparaison plus détaillée des différentes techniques, mais c'est avec plaisir que nous allons mettre en avant la puissance des neutrons. De toutes façons, les résultats de la diffusion neutronique devront, dans la mesure du possible, être complétés par ceux de la spectroscopie optique et vice versa. On peut aussi s'attendre à des synergies particulièrement intéressantes telles que la détermination de densités d'états partielles en combinant la spectroscopie neutronique et la diffusion inélastique haute résolution des rayons $\mathrm{X}$.

L'article est structuré comme suit. Nous donnons d'abord une courte introduction concernant les excitations vibrationnelles dans les solides dans le cadre de l'approximation harmonique. Nous développerons seulement les concepts les plus essentiels tels que la matrice dynamique, les vecteurs propres et les valeurs propres, les amplitudes de vibration et les densités d'états. Nous discuterons ensuite comment on peut obtenir des informations sur ces quantités vibrationnelles grâce à la diffusion inélastique des neutrons. Ceci nous amènera au facteur de structure dynamique, à la densité d'états généralisée et aux relations de dispersion. Les relaxations, et par suite la diffusion quasi élastique ne seront pas traitées explicitement ici. La discussion théorique sera complétée par une courte section sur les instruments et le traitement des données. Le " matériau " sera finalement illustré par l'exemple des vibrations externes dans les composés fullérènes.

\section{Vibrations dans les solides}

A part l'hélium tout liquide devient un solide s'il est suffisamment refroidi. Il peut soit cristalliser via une transition du premier ordre, soit devenir un verre. Dans ce dernier cas, le point en temps et température au delà duquel on considère que le système est à l'état solide est arbitraire et dépend de la durée de l'observation expérimentale et de l'historique du refroidissement. Nous ne souhaitons pas entrer plus profondément dans ce sujet complexe d'un intérêt scientifique certain, mais nous supposerons lors des considérations théoriques ultérieures que nous avons affaire à des matériaux complètement relaxés, i.e. le processus de formation est terminé et le matériaux n'évolue plus. Ceci signifie que les positions atomiques - moyennées dans le temps sur les périodes des vibrations - ne changent pas durant toute la durée de l'expérience.

\footnotetext{
${ }^{2}$ Il existe aussi des règles de sélection pour les neutrons. Mais contrairement à ce qui est affirmé dans la référence [10] -qui par ailleurs fournit une excellente revue du sujet- ceci était connu et a été exploité dès les premières expériences. Cependant du fait que l'on peut choisir librement le transfert de moment de l'événement diffusé parmi une gamme dynamique relativement large, chaque mode peut en principe être observé dans une expérience de diffusion inélastique de neutrons. Nous y reviendrons dans la section 3.4 .
} 


\subsection{Approximation adiabatique}

Pour tout solide ainsi relaxé les noyaux vibrent autour de leurs positions d'équilibre, même à zéro Kelvin par suite des oscillations quantiques. En principe le mouvement des noyaux ioniques (noyaux plus électrons de cœur) et celui des électrons de valence sont couplés ${ }^{3}$. Ce couplage provoque des phénomènes intéressants tels que la supraconductivité ou les transitions métal-isolant. En général l'approximation adiabatique [12] qui suppose que par suite de leur mobilité plus élevée -la masse des électrons est beaucoup plus faible que celle des noyaux- les électrons s'adaptent instantanément au mouvement des noyaux ioniques, marche très bien pour la plupart des matériaux. Nous voudrions néanmoins mentionner que le traitement quantique de ce concept $n$ 'est pas de tout trivial en particulier dans le cas des métaux. L'approximation adiabatique nous permet de traiter les ions comme des particules dans un potentiel extérieur. On pourrait souligner que bien qu'on étudie le mouvement des noyaux, l'information obtenue via le spectre de vibration concerne le système électronique. Après tout ce sont les électrons qui sont responsables des forces entre les noyaux.

\subsection{Modes propres harmoniques}

Dans un solide relaxé les positons atomiques moyennes sont stationnaires. Si $\mathrm{N}$ est le nombre de noyaux dans le matériau nous noterons ces $\mathrm{N}$ positions d'équilibre $\vec{r}_{k}^{0}, k=1, \ldots N$. Il est par suite possible de développer le potentiel $U\left(\vec{r}_{1} \ldots \vec{r}_{N}\right)$ en fonction des déplacements ioniques, soient $\vec{u}_{k}(t)=\vec{r}_{k}(t)-\vec{r}_{k}^{0}, k=1, \ldots N$.

$\mathrm{Si}$ on se restreint alors au second ordre on obtient :

$$
U\left(\vec{r}_{1}, \ldots \vec{r}_{N}\right)=U_{0}+\left.\sum_{\alpha, k=1}^{3, N} \frac{\partial U}{\partial u_{\alpha, k}}\right|_{0} u_{\alpha, k}+\left.\frac{1}{2} \sum_{\alpha, k=1}^{3, N} \sum_{\beta, T=1}^{3, N} \frac{\partial^{2} U}{\partial u_{\alpha, k} \partial u_{\beta, l}}\right|_{0} u_{\alpha, k} u_{\beta, l} .
$$

Les dérivées doivent être prises par rapport aux positions d'équilibre. Le premier terme dans l'équation (1) est une constante. Le second terme contient les dérivées premières du potentiel, c'est à dire les forces qui agissent sur les cours ioniques $k$ suivant la direction $\alpha$. Ces forces doivent s'annuler à l'équilibre. Le premier terme non trivial est, par suite, le terme quadratique dans l'équation (1). Si nous traitons le problème vibrationnel en nous restreignant à ce terme nous restons dans l'approximation harmonique. Cette approximation est valable tant que les déplacements restent petits. Ce que signifie " petit » dépend en réalité fortement du système étudié.

Les dérivées secondes

$$
\left.\Phi(\alpha, \beta, k, l) \equiv \frac{\partial^{2} U}{\partial u_{\alpha, k} \partial u_{\beta, l}}\right|_{0}
$$

sont les constantes de force du matériau. Elles déterminent la force de rappel que subit le noyau $k$ suivant la direction $\alpha$, quand le noyau $l$ se déplace suivant $\beta^{4}$ Les constantes de force peuvent être

${ }^{3}$ On trouve des introductions au sujet des vibrations dans les cristaux dans les livres de physique du solide de Kittel [11] et d' Ashcroft et Mermin [12]. On donne une discussion complète du sujet dans la référence [13]. Ici nous essayerons de rester aussi général que possible et c'est uniquement lorsque c'est absolument nécessaire que nous évoquerons l'ordre cristallin, i.e. l'invariance translationnelle.

${ }^{4}$ On doit porter une attention toute spéciale sur ce qui est appelé les « selftermes» $\Phi(\alpha, \beta, l, l)$. Souvent on ne peut les déterminer directement mais il faut les évaluer en utilisant la loi de Newton d'égalité de l'action et de la réaction : $\Phi_{\alpha, \beta}(l, l)=-\sum_{l \neq l} \Phi_{\alpha, \beta}\left(l, l^{\prime}\right)$. On trouvera des détails dans la référence [13] 
considérées comme les éléments d'une matrice $\mathbf{F}$ de dimensions ( $3 \mathrm{~N}$ x $3 \mathrm{~N}$ ). Comme l'opérateur d'Hamilton, dans l'approximation harmonique, contient les opérateurs moments et positions seulement jusqu'au second ordre, on peut utiliser le Théorème d'Ehrenfest [14] et traiter de façon classique le problème de trouver les vecteurs propres et les valeurs propres, i.e. qu'on a à faire à un système de $N$ particules de masses $m_{k}, k=1, \ldots N$ dans un réseau tridimensionnel connecté par des ressorts.

Les solutions de l'équation de mouvement d'un tel système ont une dépendance en temps harmonique $u_{\alpha, k}(t) \propto \exp \left(-i \omega_{j} t\right)$, i.e. il sont caractérisées par des fréquences $\omega_{j}$. Pour chaque mode de vibration d'une fréquence donnée le motif, i.e. la position et la phase d'un atome relatif aux autres, est définit par un vecteur complexe de dimension $3 \mathrm{~N}$. Ces vecteurs et fréquences sont obtenus en déterminant les vecteurs propres et les fréquences propres de la matrice dynamique

$$
D(\alpha, \beta, k, l):=\frac{\Phi(\alpha, \beta, k, l)}{\sqrt{m_{k} \cdot m_{l}}}
$$

par diagonalisation. Ou en notation matricielle

$$
\mathrm{D} \equiv \mathbf{M}^{1 / 2} \mathbf{F} \mathbf{M}^{1 / 2} \quad \text { avec } \quad \mathbf{M}^{1 / 2}(\alpha, \boldsymbol{\beta}, k, l)=\frac{1}{\sqrt{\mathrm{m}_{\mathrm{k}}}} \delta_{\alpha, \beta} \delta_{l, k} .
$$

Nous noterons les vecteurs propres à $3 \mathrm{~N}$ dimensions de la matrice dynamique par $\mathbf{e}_{\mathrm{j}}$ et les fréquences propres correspondantes par $\omega_{j}{ }^{2}$. Les vecteurs propres satisfont aux conditions d'orthonormalité et aux limites [13] :

$$
\begin{gathered}
\mathbf{e}_{j} \cdot \mathbf{e}_{j}=\sum_{\alpha, i=1}^{3, N} e_{j}(\alpha, i) e_{j^{\prime}}(\alpha, i)=\delta_{j, j^{\prime}} \\
\sum_{j=1}^{3 N} e_{j}(\alpha, i) e_{j}\left(\beta, i^{\prime}\right)=\delta_{\alpha, \beta} \delta_{i, k^{*}}
\end{gathered}
$$

En conséquence tout déplacement des noyaux peut s'exprimer comme une combinaison linéaire de ces vecteurs propres. Les vecteurs propres $\mathbf{e}_{\mathbf{j}}$ sont reliés à l'opérateur déplacement $\mathbf{u}(\alpha, l)$ des noyaux $\{l\}$ suivant les directions orthogonales $\{\alpha\}$ via la relation

$$
\mathbf{u}(\alpha, l)=\sum \sqrt{\frac{\hbar}{2 m_{l} \omega_{j}}}\left[e_{j}(\alpha, l) \mathbf{a}_{j}+e_{j}(\alpha, l) \mathbf{a}_{j}^{+}\right]
$$

où $\mathrm{a}^{+}$et a sont respectivement, les opérateurs création et annihilation des vibrations harmoniques $\{j\}$. C'est à ce point qu'on fait de nouveau la connection avec la mécanique quantique. Comme les excitations de vibration satisfont aux statistiques de Bose-Einstein, l'occupation d'un mode $\mathrm{j}$ à la température $\mathrm{T}$ est donné par

$$
\left\langle\mathbf{a}_{j}^{+} \mathbf{a}_{j}\right\rangle=n\left(\omega_{j}\right) \delta_{j, j}=\frac{1}{e^{\frac{k_{0} k^{T}}{k^{\prime}}-1}} \delta_{j, j^{n}}
$$




\section{3 Déplacements quadratiques moyens}

De l'opérateur quantique $\mathbf{u}(\alpha, 1)$ et de son adjoint $\mathbf{u}^{+}(\alpha, 1)$ on peut obtenir avec l'aide des équations 7 et 8 l'amplitude quadratique moyenne ${ }^{5}$ du particule 1 suivant la direction $\alpha$,

$$
\begin{aligned}
\overline{u(\alpha, l)^{2}} & =\left\langle\mathbf{u}(\alpha, l) \mathbf{u}^{+}(\alpha, l)\right\rangle \\
& =\sum_{j=1}^{3 N}\left|u_{j}(\alpha, l)\right|^{2}=\sum_{j=1}^{3 N} \frac{\left|e_{j}(\alpha, l)\right|^{2} \hbar}{2 m_{l} \omega_{j}}\left(2 n\left(\omega_{j}\right)+1\right)
\end{aligned}
$$

Si tous les atomes participent à un mode $j$ alors $\left|\mathrm{e}_{\mathrm{j}}(\alpha, l)\right|$ est de l'ordre de $1 /(3 \mathrm{~N})$, i.e. très petit à l'échelle macroscopique. La situation change quand les modes sont localisés. Une bonne mesure de la participation des noyaux à un mode donné $j$ est le rapport de participation [15]

$$
p_{j}=\frac{\left(\sum_{l, \alpha-1}^{3, N}\left|u_{j}(\alpha, l)\right|^{2}\right)^{2}}{N \sum_{l, \alpha-1}^{3, N}\left|u_{j}(\alpha, l)\right|^{4}} .
$$

Pour un déplacement rigide de l'ensemble du système dans la direction $\alpha$ on a $\mathrm{u}_{\mathrm{j}}(\alpha, l) \sim 1 / \mathrm{N}=$ constante pour tous les atomes $l$, et par suite $\mathrm{p}_{\mathrm{i}}=\left(\Sigma_{\mathrm{l}, \mathrm{a}} 1\right)^{2} / \mathrm{N}\left(\left(\Sigma_{\mathrm{l}, \mathrm{a}} 1\right)=1\right.$. Pour des modes étendus, $\mathrm{p}_{\mathrm{j}}$ reste proche de 1 , tandis que pour des modes très localisés, $\mathrm{p}_{\mathrm{j}} \sim \mathrm{N}_{\text {part }} / \mathrm{N}$, où $\mathrm{N}_{\text {part }}$ correspond au nombre des atomes participants. Ce dernier résultat est facile à démontrer dans le cas d'un déplacement rigide d'un cluster de particules de $\mathrm{N}_{\text {part }}$ atomes.

En moyennant l'équation 9 sur toutes les direction nous obtenons

$$
\overrightarrow{u_{i}^{2}}=\frac{1}{3}\left\langle\overrightarrow{\mathbf{u}}, \overrightarrow{\mathbf{u}}_{l}^{+}\right\rangle=\frac{1}{3} \sum_{\alpha=1}^{3} \sum_{j=1}^{3 N} \frac{\left.e_{j}(\alpha, l)\right|^{2} \hbar}{2 m_{l} \omega_{j}}\left(2 n\left(\omega_{j}\right)+1\right)
$$

et finalement en moyennant l'équation 11 sur tous les noyaux on obtient le déplacement carré moyen

$$
\overline{u^{2}}=\frac{1}{3 N} \sum_{l=1}^{N}\left\langle\overrightarrow{\mathbf{u}_{l}}, \overrightarrow{\mathbf{u}}_{l}^{+}\right\rangle=\frac{1}{3 N} \sum_{\alpha=1}^{3} \sum_{l=1}^{N} \sum_{j=1}^{3 N} \frac{\left|e_{j}(\alpha, l)\right|^{2} \hbar}{2 m_{l} \omega_{j}}\left(2 n\left(\omega_{j}\right)+1\right)
$$

Si tous les noyaux ont la même masse on peut à nouveau simplifier l'expression ci-dessus à l'aide de la relation d'orthogonalité (5):

$$
\overline{u^{2}}=\left\langle\overrightarrow{\mathbf{u}}_{l} \overrightarrow{\mathbf{u}}_{i}^{+}\right\rangle=\frac{1}{3 N} \sum_{j=1}^{3 N} \frac{\hbar}{2 m_{l} \omega_{j}}\left(2 n\left(\omega_{j}\right)+1\right)
$$

Le déplacement carré moyen est une quantité très importante car elle est à la fois intuitive et détermine de nombreuses propriétés du matériau. En particulier un accroissement de $\bar{u}$ en fonction de la température plus important que ce qui est indiqué par l'équation 13 indique une faillite de l'approximation harmonique et peut être soit un précurseur d'une transition de phase soit un indicateur d'un phénomène de relaxation.

\footnotetext{
${ }^{5}$ Il faut se rappeler cela et que $\left\langle\mathbf{a}_{\mathrm{j}} \mathbf{a}_{\mathrm{j}}\right\rangle=\left\langle\mathbf{a}_{\mathrm{j}}^{+} \mathbf{a}_{\mathrm{j}}^{+}\right\rangle=0$. On écrit les opérateurs déplacements en caractères gras et leur valeurs moyensen lettres normales.
} 
L'équation 13 peut s'écrire d'une manière plus élégante en introduisant la densité-d'état-devibration, i.e. le nombre de modes vibrationnels par intervalle d'énergie $d E$.

$$
G(\omega) \equiv \frac{1}{3 N} \sum_{j=1}^{3 N} \delta\left(\omega-\omega_{j}\right)
$$

La normalisation a été choisie de telle manière que

$$
\int_{0}^{\infty} G(\omega) d \omega=1
$$

Dans la figure 1 nous montrons l'exemple de la densité d'états d'un polymère fullerène dopé. En portant l'éq. 14 dans l'éq. 15 nous obtenons:

$$
\overline{u^{2}}=\frac{\hbar}{2 m} \int_{0}^{\infty} \frac{G(\omega)}{\omega}(2 n(\omega)+1) d \omega
$$
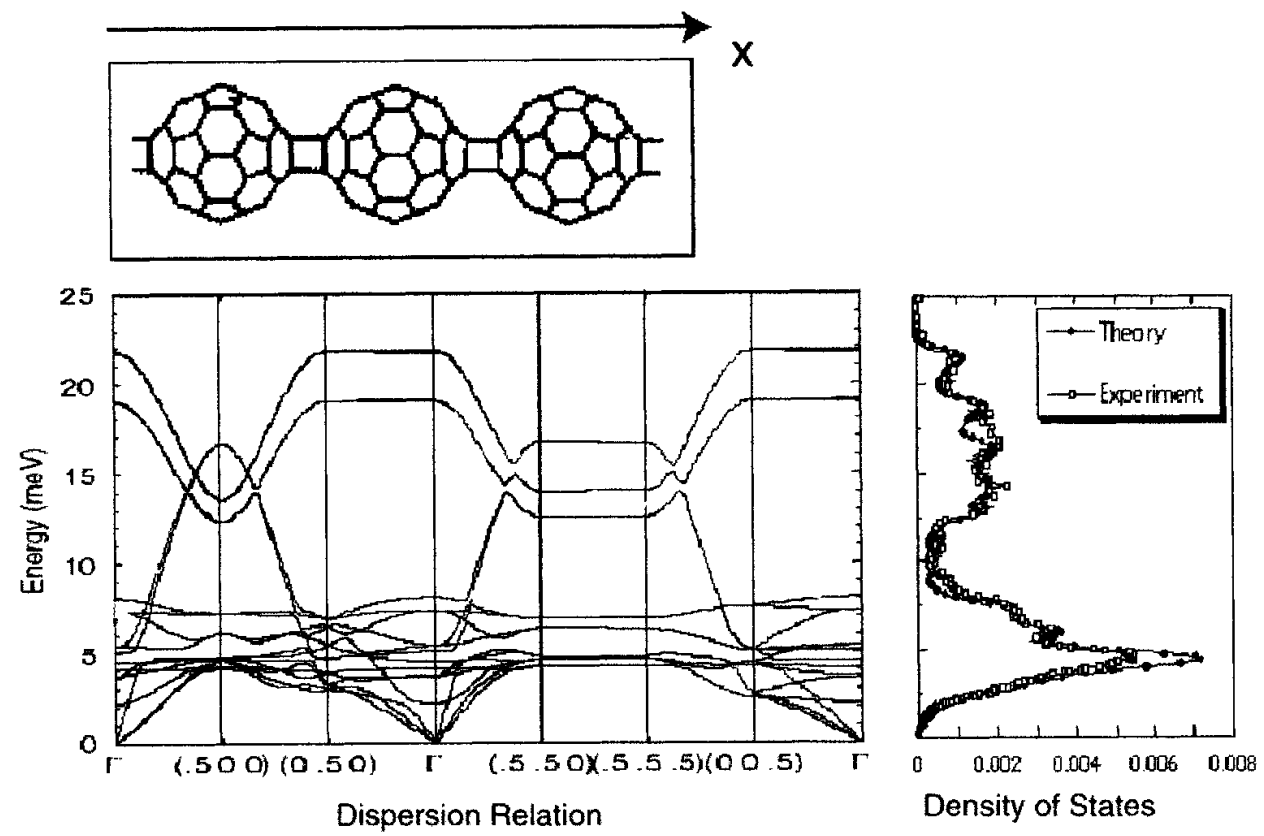

Figure 1: Relations de dispersion de $\mathrm{RbC}_{60}$ dans la phase polymère linéaire. Il y a deux unités $\mathrm{RbC}_{60}$ dans la cellule unitaire ce qui mène à 18 modes externes, i.e. des modes qui ne déforment pas le $\mathrm{C}_{60}$ (La molécule $\mathrm{C}_{60}$ possède six degrés de liberté externe : trois translation et trois rotations). Les chaînes de polymères sont orientées suivant l'axe des $x$. Le caractère $1 D$ du système est évident à partir de la forte dispersion de la branche acoustique (LA) suivant $k_{\mathrm{x}}$ tandis que la pente du mode LA est comparable à celle du monomère pour $\mathrm{k}_{\mathrm{y}}$ et $\mathrm{k}_{\mathrm{z}}$. Les branches les plus élevées des modes externes cortespondent à des mouvements de libration autour d'axes perpendiculaires à la direction des chaînes tandis que les fréquences des modes de torsion de la chaîne elle-même sont prédits à des valeurs $\sim 4 \mathrm{meV}$ qui sont étonnamment basses. Pour plus de détails voir la section 5 .

Dans un système composé de plusieurs espèces atomiques, ce qui intéresse le scientifique diffuseur de neutron c'est la densité d'états partielle définie par

$$
F_{\kappa}(\omega):=\frac{1}{3 N_{x}} \sum_{j=1}^{3 N} \sum_{t \in\left\{x_{j}\right\}}\left|\vec{e}_{j}(l)\right|^{2} \delta\left(\omega-\omega_{j}\right)
$$


La somme portant sur l'indice $l$ inclut l'ensemble des $N_{\kappa}$ atomes appartenant à l'espèce $\kappa$. En partant de $\mathrm{F}_{\kappa}(\omega)$ il est possible de déterminer les facteurs Debye-Waller et les sections efficaces de diffusion (voir section 3.2)

\subsection{Les phonons dans les systèmes avec invariance de translation}

Jusqu'à présent dans notre discussion nous n'avons pas fait allusion à l'invariance de translation. Nous devons maintenant discuter du cas important des vibrations dans les cristaux. Comme le groupe des translations est Abélien les représentations irréductibles sont à une dimension. Dans différentes cellules unités $\mathrm{k}$ et $\mathrm{l}$, la fonction d'onde peut seulement différer par un facteur de phase et par suite, a la forme

$$
\exp \left(\overrightarrow{i q} \cdot\left(\vec{r}_{k}^{\mathrm{o}}-\vec{r}_{l}^{\mathrm{o}}\right)\right)
$$

avec $\vec{q}$ dans la première zone de Brillouin du réseau réciproque [16] $]_{k}^{6}$ dénote le vecteur reliant l'origine à la cellule $\mathrm{k}$ de la maille. Nous utiliserons ce résultat pour faire un développement des déplacements sous la forme

$$
u_{\alpha}(l, \kappa)=\sum_{j, q} \sqrt{\frac{\hbar}{2 N m_{\kappa} \omega_{j}(\vec{q})}}\left[e_{j}(\alpha, \kappa \mid \vec{q}) e^{i \vec{q} \overrightarrow{\hat{q}}_{j}^{0}} a_{j}(\vec{q})+e_{j}^{*}(\alpha, \kappa \mid \vec{q}) e^{i \vec{q} \cdot \vec{r}_{\vec{j}}^{0}} a_{j}(\vec{q})\right]
$$

L'index $\kappa$ permet de différencier parmi les atomes dans la cellule primitive. La principale différence par rapport à l'équation 7 est que dans le cristal on a à faire avec une classification ${ }^{7}$ des $3 \mathrm{~N}$ vecteurs propres en termes d'ondes planes.

Si on porte cette expression dans opérateur de Hamilton $\mathrm{H}$ du système celui-ci s'écrit comme

$$
\mathbf{H}=\sum_{j, \bar{q}} \hbar \omega_{j}(\vec{q})\left\{a_{j}^{+}(\vec{q}) a_{j}(\vec{q})+\frac{1}{2}\right\}
$$

pourvu que la relation

$$
\omega_{j}^{2}(\vec{q}) e_{j}(\vec{q})=\mathbf{D}(\vec{q}) e_{j}(\vec{q})
$$

soit respectée, avec la matrice dynamique dans l'espace réciproque

$$
D_{\alpha, \beta, \kappa, \kappa^{\prime}}(\vec{q})=\sum_{k} \frac{1}{\sqrt{m_{k}}} \frac{\partial^{2} U}{\partial u_{\alpha}(l=0, \kappa) \partial u_{\beta}\left(k, \kappa^{\prime}\right)} \frac{1}{\sqrt{m_{k^{\prime}}}} e^{i \vec{q} \cdot\left(\bar{r}_{i=0}^{0}-\bar{k}_{k}^{0}\right)}
$$

Il y a 3 r vecteurs propres pour chaque $\vec{q}$ de la première zone de Brillouin, avec $\mathrm{r}$ désignant le nombre d'atomes dans la cellule primitive du cristal et $\vec{q}$ un bon nombre quantique. Le vecteur $\mathbf{e}_{\mathrm{j}}(\vec{q})$ de dimension $3 \mathrm{r}$ décrit le motif de la vibration dans la cellule primitive. En conséquence, on

\footnotetext{
${ }^{6}$ Ce résultat est généralement valable. Cependant, en dehors de l'approximation harmonique il ne simplifie pas réellement la tache de trouver les solutions de l'Hamiltonien multiparticule.

${ }^{7}$ Une classification ultérieure peut s'obtenir en exploitant l'ensemble de la symétrie du cristal, pourvu que l'étoile du vecteur $\vec{q}$ ait une dimension inférieure à la dimension du groupe ponctuel, i.e. pourvu que $\vec{q}$ soit replié sur lui-mêtme par action d'autres éléments de symétrie que l'opération identité [16]
} 
opère avec des feuillets de dispersion $\omega_{\mathrm{j}}(\vec{q})$ dans l'espace réciproque ${ }^{8}$. Comme ces feuillets sont périodiques dans l'espace réciproque, l'information complète concernant les vibrations est contenue dans la première zone de Brillouin. La figure 1 donne un exemple de relations de dispersion dans le cas des fullerènes.

Une conséquence immédiate de l'invariance de translation est l'absence de modes localisés. Dans un cristal les vibrations s'étendent toujours sur l'ensemble du volume. Ceci ne signifie pas que le rapport de participation doive être égal à un car une partie des atomes de la cellule primitive peut ne pas participer à une vibration donnée. Cependant le rapport de participation n'est jamais inférieur à $1 / r$. Le fait que $\vec{q}$ soit un bon nombre quantique dans le cas d'un cristal a des implications profondes pour ses propriétés thermodynamiques et de transport. Ceci influence aussi fortement le processus de diffusion comme on le verra dans la section 3.4.

\section{Les informations vibrationnelles testées par les neutrons}

La puissance de la diffusion des neutrons réside dans sa simplicité. Bien que la diffusion des neutron soit souvent présentée comme une technique expérimentale lourde du fait qu'elle requiert un équipement complexe et, par suite coûteux, il est actuellement bien difficile de concevoir un évènement de diffusion plus simple que celui impliquant des neutrons froids ou thermiques. Pourquoi est-ce ainsi? Dû au fait que l'interaction entre noyaux et neutron s'exerçe à courte distance -quelques fm- par rapport à la longueur d'onde du neutron - quelques $\AA$ - la diffusion est isotrope, i.e. seules des ondes-s sont requises dans le développement de la fonction d'onde diffusée [14]. A cause de cette isotropie on peut décrire la diffusion par un scalaire qui est l'amplitude de diffusion $b$. La partie complexe de $b$ prend en compte l'absorption. Comme la diffusion est généralement faible le flux de neutrons incident peut être considéré comme constant et les événements de diffusion multiple ${ }^{9}$ sont rares pour des volumes diffusant raisonnables ${ }^{10}$. Ceci a pour conséquence qu'on peut travailler avec le plus petit ordre de perturbation i.e. avec l'approximation de Born ${ }^{11}$.

\subsection{Facteur de structure dynamique}

Dans une expérience de diffusion inélastique des neutrons on détermine à la fois les changements en direction et en énergie des neutrons. Par suite, on s'intéresse à la double différentielle de la section efficace, i.e. au nombre de neutrons avec un vecteur $k_{\mathrm{i}}$, un spin $\sigma_{\mathrm{i}}$ et une énergie $\mathrm{E}_{\mathrm{i}}$ qui sont

\footnotetext{
${ }^{8}$ Les feuillets de dispersion sont des objets tri-dimensionnels (ou plans) dans l'espace $(\vec{Q}, \omega)$ à 4 dimensions. Les nombres quantiques de ces modes propres se situent tous sur ces objets.

${ }^{9}$ Nous reviendrons sur ce point plus tard.

${ }^{10}$ Dans toutes les expériences on devrait prendre comme règle d'or de travailler avec des volumes d'échantillons tels qu'au maximum $10 \%$ des neutrons sont diffusés alors que $90 \%$ traversent sans perturbation dans la direction avant. Ce « gaspillage » de neutrons est indispensable pour obtenir des résultats propres et d'interprétation facile.

11 L'approximation de Born est en défaut pour une diffusion fortement concentrée suivant des angles solides particulier. Un exemple typique d'une telle diffusion est la diffusion de Bragg dans des cristaux très purs. Dans ce cas on a à faire au probleme d'extinction. On peut traiter l'extinction uniquement dans le cadre de la théorie de la diffusion dynamique qui prend en compte une superposition cohérente des fonctions d'onde incidentes et diffusées[17]. Il semble exclus que l'extinction puisse s'observer pour des signaux inélastiques même avec le silicium de pureté maximale.
} 
diffusés dans l'angle solide $d \Omega$ et dont l'énergie finale est dans l'intervalle $E_{f} \ldots E_{f}+d E_{f}$ et le spin $\sigma_{f}$. Dans l'approximation de Born cette section efficace est donnée par

$$
\frac{d^{2} \sigma}{d \Omega d E_{f}}=\frac{k_{f}}{k_{i}} \sum_{\lambda_{i} \lambda_{f}} p_{\lambda_{i}}\left\langle\left.\left(\vec{k}_{f} \sigma_{f} ; \lambda_{f}|V| \vec{k}_{i} \sigma_{i} ; \lambda_{i}\right)\right|^{2} \delta\left(\hbar \omega+E_{\lambda_{i}}-E_{\lambda_{f}}\right)\right.
$$

Avec $\lambda_{i}$ et $\lambda_{f}$ désignent les états initial et final de l'échantillon. Le paramètre $p_{\lambda_{i}}$ pondère les états initiaux et contient par exemple les facteurs de température décrivant l'occupation thermique de ces états. Le préfacteur $k_{f} / k_{i}$ a son origine dans la normalisation relative au flux incident et à la densité $p_{\lambda_{f}}\left(E_{f}\right)=m k_{f} / \hbar^{2}$ des états finaux accessibles aux neutrons diffusés. La fonction $\delta$ prend en compte la conservation de l'énergie. Le seul potentiel pour lequel l'approximation de Born donne une diffusion isotrope est le pseudo potentiel de Fermi

$$
V(\vec{r})=\frac{2 \pi \hbar^{2}}{m_{n}} \sum b_{l} \delta\left(\vec{r}-\vec{r}_{l}\right) ; l=1 \ldots N,
$$

avec à nouveau $\vec{r}_{l}$ indiquant la position du $l$-ième noyau ${ }^{12}$. Utilisant ce potentiel dans l'équation 20 nous amène à ce qu'on appelle l'équation maîtresse de la diffusion inélastique des neutrons :

$$
\frac{d^{2} \sigma}{d \Omega d E_{f}}=\frac{1}{N} \frac{k_{f}}{k_{i}} \sum_{\lambda_{i} \sigma_{i}} p_{\lambda_{i}} p_{\sigma_{i}} \sum_{\lambda_{f}, \sigma_{f}}\left|<\sigma_{f} ; \lambda_{f}\right| \sum b e^{i \bar{Q} \cdot \bar{r}_{1}}\left|\sigma_{i} ; \lambda_{i}>\right|^{2} \delta\left(\hbar \omega+E_{\lambda_{i}}-E_{\lambda_{f}}\right),
$$

avec $\vec{Q}=\vec{k}_{i}-\vec{k}_{f}$. On devra continuer à garder explicitement les états de spin des neutrons toutes les fois que les longueurs de diffusion $b^{(+)}$et $b^{(-)}$sont différentes ${ }^{13}$. Il est possible d'écrire la double différentielle de la section efficace sous la forme

$$
\frac{d^{2} \sigma}{d \Omega d E_{f}}=\frac{k_{f}}{k_{i}} N<\overline{b^{2}}>S(\vec{Q}, \omega)
$$

avec $\left\langle\overline{b^{2}}\right\rangle=N^{-1} \sum_{i} \overline{b_{i}^{2}}$ est telle que la barre sur la longueur de liaison $b_{i}$ dénote une moyenne sur le spin et les isotopes ${ }^{14}$. La fonction de diffusion ainsi définie $s(\vec{Q}, \omega)$, a les dimensions de l'inverse d'une énergie et ne contient pas de variables qui tiennent compte des fonctions d'onde des neutrons incidents et de ceux diffusés. L'équation 23 illustre donc le fait que nous pouvons découpler la sonde du système étudié. Ceci est une autre caractéristique importante de l'INS qui a son origine dans le fait que les neutrons, comme ce sont des sondes très faibles, étudient un système non perturbé. En d'autres termes, l'expérience fournit une information directe sur les fluctuations

\footnotetext{
${ }^{12}$ Notez le fait que la fonction $\delta$ a les dimensions de l'inverse d'un volume. En outre $b_{l}$ est, comme on l'a déjà indiqué, une longueur de diffusion. La section efficace de diffusion totale pour un diffuseur isolé est donc: $\sigma=4 \pi|b|^{2} . m_{n}$ est la masse du neutron.

${ }^{13}$ En sommant sur l'état des spins au final nous supposons que les neutrons sont détectés indépendamment de leur spin, i.e. sans analyse de leur polarisation. $p_{a}$ donne la distribution des spins du faisceau incident. Les étiquettes $(+)$ et $(-)$ se réfèrent aux spins notés $I+1 / 2$ et $I-1 / 2$ impliqués dans la diffusion du neutron par un noyau de spin total $I$.

${ }^{14}$ Dans la suite nous supposerons que les longueurs de diffusion sont réelles, i.e. nous négligerons l'absorption
} 
spontanées dans l'échantillon, et cela en dépit du fait que les neutrons interagissent avec lui ${ }^{15}$. Mathématiquement cette situation s'exprime par le théorème de fluctuation-dissipation [25].

En exprimant la fonction $\delta$ dans l'équation 22 comme une intégrale sur le temps et en utilisant la représentation d'Heisenberg de la mécanique quantique on obtient pour la fonction de diffusion ${ }^{16}$

$$
S(\vec{Q}, \omega)=\frac{1}{2 \pi \hbar N<\overline{b^{2}}>} \int_{-\infty}^{\infty} d t e^{-i \omega x} \sum_{k t}^{N} \overline{b_{k} b_{l}}<e^{-i \vec{Q} \cdot \vec{k}_{k}} e^{-i \vec{Q} \cdot \bar{r}_{l}(t)}>.
$$

$S(\vec{Q}, \omega)$ est appelé le facteur de structure dynamique ${ }^{17}$.

Maintenant il nous faut déterminer le facteur de structure dynamique pour des vibrations harmoniques. A cette fin nous utiliserons l'identité de Bloch pour convertir la valeur moyenne des fonctions exponentielles apparaissant dans l'équation 24 en exponentielles de valeurs moyennes $[25]^{18}$.

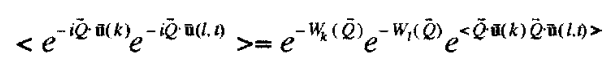

le facteur Debye Waller étant défini comme

$$
\left.W_{k}(\vec{Q})=\frac{1}{2}<[\vec{Q} \cdot \overrightarrow{\mathbf{u}}(\mathbf{k})]^{2}\right\rangle
$$

La fonction exponentielle dans l'équation 25 peut maintenant se développer en termes de

$$
<\vec{Q} \cdot \overrightarrow{\mathbf{u}}(k) \vec{Q} \cdot \overrightarrow{\mathbf{u}}(l ; \mathrm{t})>
$$

Les différents termes dans le développement se réfèrent respectivement, au canal élastique, aux phonons du premier ordre, du second ordre et d'ordre supérieur. Pour le moment concentrons nous sur la contribution à un phonon à $S(\vec{Q}, \omega)$. Portant l'expression de l'équation 7 pour l'opérateur $\overrightarrow{\mathbf{u}}$ dans l'équation 25 on obtient pour les phonons du premier ordre

15 La situation est analogue à l'étude d'un pendule classique piloté de l'extérieur pour de faibles amplitudes d'oscillation. C'est seulement pour un très petit intervalle des fréquences de commande $\omega$, centré autour de la fréquence propre $\omega_{0}$ que l'on observera la résonance. Comme la fréquence de résonance est indépendante de la force de commande, grâce à cette expérience on étudie les propriétés intrinsèques du système non perturbé.

${ }^{16}$ Nous ne détaillerons pas la procédure ici et renverrons le lecteur à la littérature [25]

${ }^{17}$ Comme on le voit $S(\vec{Q}, \omega)$ contient les longueurs de diffusion $b_{l}$. Le découplage de la sonde, i.e. des neutrons, et de l'échantillon est, par conséquent, incomplet. C'est seulement pour un système caractérisé par une seule longueur de diffusion $S(\vec{Q}, \omega)$ devient une quantité exclusivement déterminée par les fluctuations de l'échantillon. Ceci est particulièrement commode car dans ce cas $S(\vec{Q}, \omega)$ peut être calculé à partir de la théorie de la réponse linéaire sans faire référence à l'expérience. Nous verrons plus tard que le fait que les longueurs de diffusion entrent dans $s(\vec{Q}, \omega)$ complique l'obtention d'une information spécifique à l'échantillon à partir des données de diffusion inélastique.

18 Notez aussi que l'identité de Bloch est valable seulement pour des opérateurs de Bose. Par suite, c'est valable uniquement dans l'approximation harmonique, même si à ce stade de la discussion on ne s'appuie pas explicitement sur ce fait. 


$$
S(\vec{Q}, \omega)=\frac{1}{2 N<\overline{b^{2}}>} \sum_{j} \frac{\left|F_{j}(\vec{Q})\right|^{2}}{\omega_{j}}\left[\left(n_{j}+1\right) \delta\left(\omega-\omega_{j}\right)+n_{j} \delta\left(\omega+\omega_{j}\right)\right]
$$

avec

$$
\left|F_{j}(\vec{Q})\right|^{2}=\sum_{k T}^{N} \frac{\overrightarrow{b_{k} b_{l}}}{\sqrt{m_{k} m_{l}}} e^{-W_{k}(\tilde{Q})-W_{i}(\bar{Q})}\left(\vec{Q} \cdot \vec{e}_{j}(k)\right)\left(\vec{Q} \cdot \vec{e}_{j}(l)\right) e^{\bar{Q} \cdot\left(\bar{c}_{k}-\bar{r}_{l}\right)}
$$

L'expression $|\mathrm{F}(\mathrm{Q})|^{2}$ illustre le facteur de structure pour les vibrations $\{j\} . \vec{e}_{i}(k)$ est la composante tridimensionnelle de $\mathbf{e}_{j}$ vecteur propre à $3 \mathrm{~N}$-dimensions du mode $\mathrm{j}$, relatif à l'atome $\mathrm{k}$. Le facteur Debye-Waller peut alors se calculer comme étant

$$
W_{k}(Q)=\frac{1}{2}\left\langle\left(\vec{Q} \cdot \overrightarrow{\mathbf{u}}_{\mathrm{k}}\right)^{2}\right\rangle=\frac{\hbar}{m_{i}} \sum_{j} \frac{\left(\vec{Q} \cdot e_{j}(k)\right)^{2}}{\omega_{j}}\left(2 n_{j}+1\right)
$$

Nous devons maintenant faire quelques remarques concernant les expressions obtenues :

1. Comme nous l'avons déjà mentionné, les neutrons sondent les fluctuations dans l'échantillon. La diffusion inélastique a lieu à condition que l'évolution en espace et temps d'une fluctuation soit compatible avec le motif en espace et temps fourni par la superposition des fonctions d'onde des neutrons incident et émergent. Ceci est à la base du facteur de structure dynamique dans l'équation 28.

2. Comme les neutrons sondent les fluctuations, l'intensité de la diffusion est déterminée par le carré de l'amplitude des déplacements atomiques. Dans un système harmonique les déplacements carrés moyens sont obtenus via l'équation 12 . On peut aisément vérifier que les quantités qui déterminent le déplacement carré moyen, i.e. l'inverse des fréquences $\omega_{\mathrm{j}}{ }^{-1}$, l'inverse des masses $\mathrm{m}^{-1}$ et les facteurs de température $n\left(\omega_{\mathrm{j}}\right)$ figurent toutes dans le facteur de structure dynamique.

3. Comme la diffusion est déterminée par des amplitudes au carré on peut immédiatement en déduire que l'intensité dépendra de la température via le facteur d'occupation $n(\omega)+1$ donné dans l'équation 8. $n(\omega)+1$ apparaît d'ailleurs explicitement dans l'équation 28. Mais comme les facteurs Debye-Waller ne sont pas indépendants de la température $(n(\omega)$ $+1)$ ne rend pas compte de toute la variation en température du facteur de structure. En dépit de ce fait il est très utile d'écrire la fonction de diffusion sous la forme

$$
S(\vec{Q}, \omega)=\frac{1}{\pi}\{1+n(\omega)\} \chi^{\prime \prime}[\vec{Q}, \omega]
$$

A cause du théorème de fluctuation-dissipation la fonction $\chi^{\prime \prime}[\vec{Q}, \omega]$ doit être identifiée avec la susceptibilité généralisée du système, i.e. avec la réponse linéaire du système à une perturbation périodique de fréquence $\omega$. L'adjective généralisée nous indique que la réponse correspond à une perturbation qui se couple au système via la longueur de diffusion du neutron. Pour un système à une composante elle est identique à la susceptibilité réelle. Pour un oscillateur harmonique simple la susceptibilité est 


$$
\begin{aligned}
\chi^{\prime \prime}[\omega] & =\frac{1+n(\omega)}{2 m \omega_{0}}\left\{\delta\left(\omega-\omega_{0}\right)-\delta\left(\omega+\omega_{0}\right)\right\} \\
& =\frac{1}{2 m \omega_{0}}\left\{\left[1+n\left(\omega_{0}\right)\right] \delta\left(\omega-\omega_{0}\right)+n\left(\omega_{0}\right) \delta\left(\omega+\omega_{0}\right)\right\}
\end{aligned}
$$

par suite, pour un ensemble d'oscillateurs harmoniques non couplés $\chi$ '' $[\omega]$ est indépendant de la température. L'équation 32 est compatible avec la formule plus générale qu'on peut déduire pour $\chi$ '' $[\omega]$ à partir de l'équation 28 .

4. Si on néglige les effets d'interférence dans l'équation 29 , alors la diffusion à un-phonon d'une part croît avec $Q^{2}$ et d'autre part décroît en $e^{-W(\bar{Q})}$. Par suite, il est préférable de réaliser les expériences avec des transferts de moments importants tant que le facteur de Debye-Waller n'est pas trop grand, i.e. pour des déplacements quadratiques moyens petits ou de basses températures. Les effets d'interférence donnent aux intensités des phonons une dépendance en $\vec{Q}$ très compliquée. En déterminant cette dépendance il est en principe possible d'obtenir des informations sur le motif des vibrations, i.e. sur les vecteurs propres, qui par l'intermédiaire de l'équation 29 déterminent les effets d'interférence. Dans la pratique cette procédure est assez complexe nécessitant des calculs de dynamique de réseau très performants. Si la symétrie du système est élevée elle peut contraindre les vecteur propres de certaines vibrations de manière à rendre leur détermination possible sans calculs

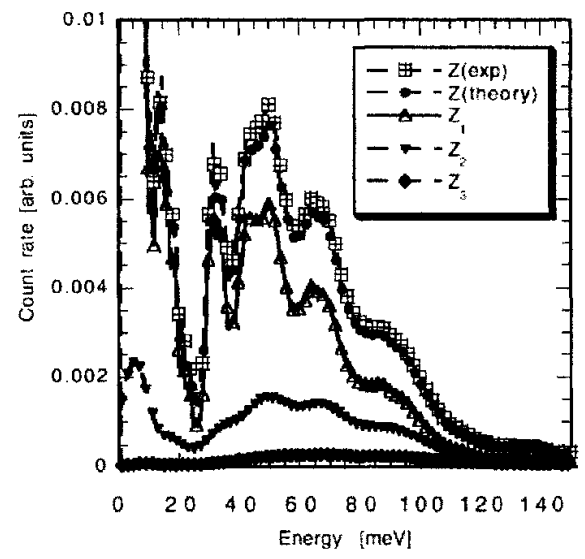

Figure 2: Spectre inélastique de $\mathrm{RbC}_{60}$ à 350 $\mathrm{K}(\mathrm{Ei}=4.75 \mathrm{meV})$ après sommation sur tous les détecteurs et conversion en énergie [6]. Z(exp): resultants expérimentaux ; $Z$ (théorie) : spectre calculé en utilisant l'expression self-consistante $\mathbf{G}(\omega)$ comme entrée $; Z_{n}, n=1,2,3$ contribution d'ordre au spectre calculé. Le déplacement carré moyen est $\left.<u^{2}\right\rangle=0.038 \AA^{2}$.

5. Les fonctions- $\delta$ expriment la conservation de l'énergie. Dans le cas de diffusion en perte d'énergie le neutron perd de l'énergie qui est récupérée par l'échantillon sous forme de vibrations. Si la diffusion en perte d'énergie est possible à toutes températures, elle requiert néanmoins que l'énergie du neutron soit supérieure à l'énergie des vibrations qu'on veut sonder. La diffusion en gain d'énergie implique un transfert d'énergie de l'échantillon au neutron et est négligeable aux températures où $k_{B} T \ll \hbar \omega$. 
6. Les termes de phonons d'ordres plus élevés croissent à la fois avec la température $T$ et avec le transfert de moment $Q$ (pour un exemple de contributions multi-phonon voir la Fig. 2). Ils correspondent à la création et à l'annihilation simultanée de plus d'une vibration durant un événement de diffusion. Des événements mixtes (avec simultanément création de $n$ et annihilation de $m$ vibrations) sont également possibles. On peut montrer que les changements de la diffusion en fonction de la température induits par le facteur Debye-Waller et les termes de phonons d'ordres supérieurs se compensent les uns les autres, de sorte que l'on trouve que la diffusion augmente en gros avec le facteur d'occupation. Dans de bons systèmes harmoniques on peut vérifier cette affirmation sur un intervalle de plusieurs centaines de degrés Kelvin (voir Fig. 3). Le spectre de diffusion des phonons d'ordre élevé est moins structuré que celui du processus à un-phonon. En conséquence, il constitue un bruit de fond étendu qui influence la distribution d'intensité mais altère rarement la position des pics [6].

7. Le facteur de structure dans l'équation 28 contient une somme double sur les indices atomiques. Comme les états de spin ou isotopiques des différents noyaux ne sont généralement pas corrélés $\bar{b}_{k} b_{i}=\bar{b}_{k} \cdot \bar{b}_{i}$ pour $\mathrm{k} \neq 1$. Par suite, il est opportun de séparer cette somme en deux contributions qui sont appelées cohérente et incohérente, respectivement :

8.

$$
\left|F_{j}(\vec{Q})\right|_{\text {incoh }}^{2}=\sum_{k}^{N} \frac{\overline{b_{k}^{2}}-\overline{b_{k}^{2}}}{m_{k}} e^{-2 W_{k}(\bar{Q})}\left|\vec{Q} \cdot \vec{e}_{j}(k)\right|^{2}
$$

et

$$
\left|F_{j}(\vec{Q})\right|_{\mathrm{coh}}^{2}=\left|\sum_{k=1}^{N} \frac{\overline{b_{k}}}{\sqrt{m_{k}}} e^{-2 w_{k}(\bar{Q})}\left(\vec{Q} \cdot \vec{e}_{j}(k)\right) e^{i \vec{Q} \vec{R}_{k}}\right|^{2}
$$

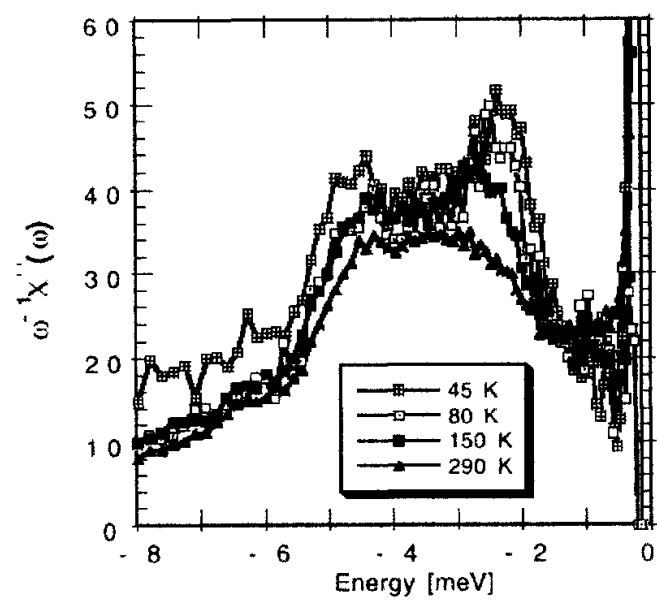

Figure 3: Evolution de $\omega^{-1} \chi^{\prime}(\omega)$ (moyenné sur tous les angles de diffusion accessibles) pour l'état polymère de $\mathrm{Rb}_{1} \mathrm{C}_{60}$ dans la région basse temperature. Comme on s'y attend pour un système harmonique, la susceptibilité généralisée est invariante à part des changements mineurs dans la région $1.5 \mathrm{meV}<\hbar \omega<3 \mathrm{meV}$. $\omega^{-1} \chi^{\prime \prime}(\omega)$ a été choisi au lieu de $\chi^{\prime \prime}(\omega)$ parce qu'il est proportionnel à $S(\omega)$ pour $\hbar \omega \ll k_{b} T$ et ainsi garantit la statistique des données. 

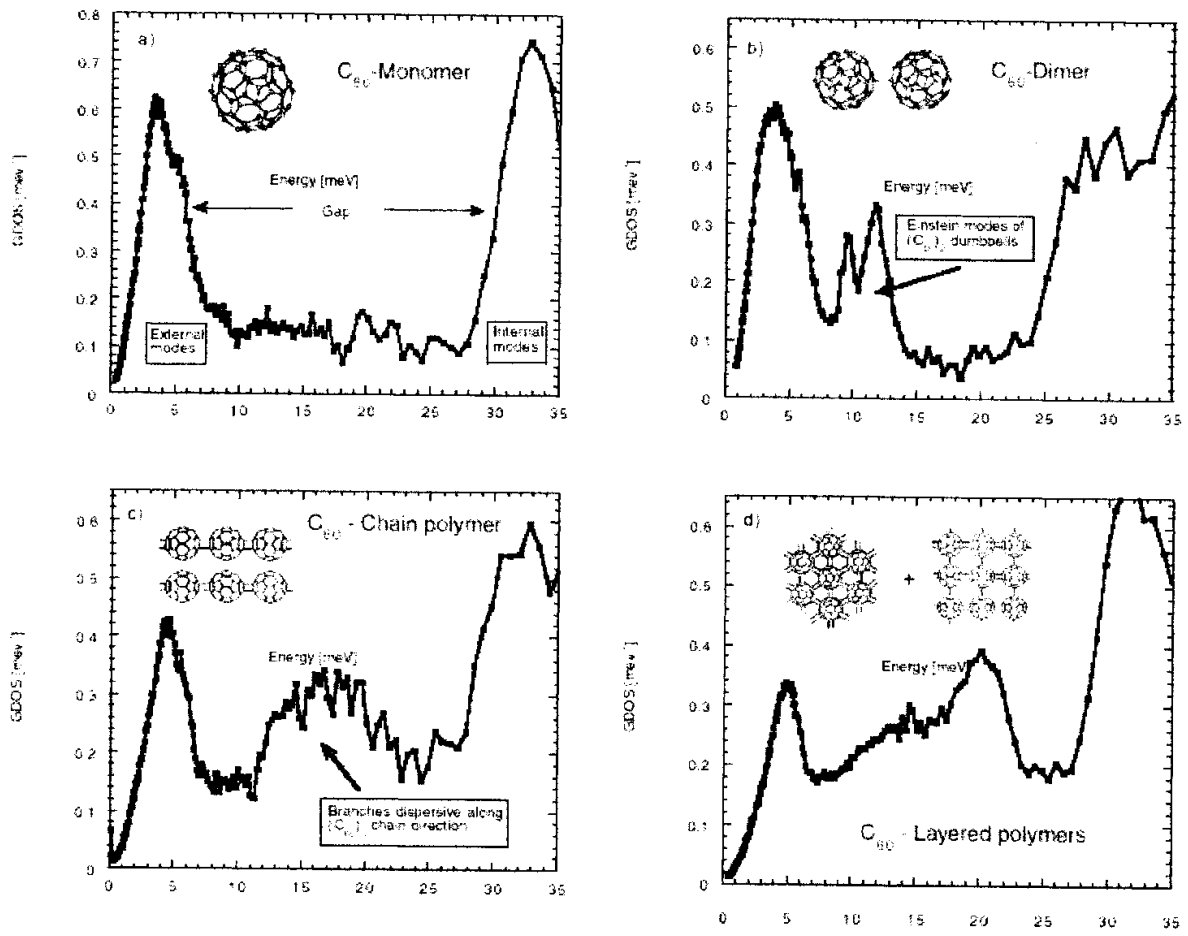

Figure 4: $G(\omega)$ s'obtient à partir du spectre de diffusion inélastique en utilisant l'instrument à temps de vol neutrons froids IN6 de l'ILL. a)- $\mathrm{C}_{60}$ à $300 \mathrm{~K}$ dans la phase plastique b)- $\left(\mathrm{C}_{60}\right)_{2}$ dimère de $\mathrm{RbC}_{60} \quad$ c)-chaines linéaires de $\mathrm{C}_{60}$ polymérisé sous pression [28] d)- feuillets de $C_{60}$ polymère 2-D polymérisé sous pression.

\subsection{Densité d'états vibrationnels}

Comme on l'a déjà exposé brièvement dans la section 2.3 la densité d'états vibrationnels $G(\omega)$ est une quantité particulièrement utile pour l'étude des vibrations. La figure 4 montre des exemples pour le spectre des modes externes des fullérènes $\mathrm{C}_{60}$.

Dans un système à une composante atomique $G(\omega)$ est directement reliée à la partie à un seul phonon de la fonction de diffusion incohérente [25] définie ci-dessus.

$$
\left(\frac{d^{2} \sigma}{d E_{f} d \Omega}\right)_{\mathrm{inc}}=\frac{N \sigma_{i}}{8 \pi m} \frac{k_{f}}{k_{i}} Q^{2} e^{-2 \omega(\bar{Q})} \frac{G(\omega)}{\omega}(n(\omega)+1),
$$


avec $\sigma_{i} \equiv 4 \pi\left(\bar{b}_{i}^{2}-\vec{b}_{i}^{2}\right)$ signifiant la section efficace de diffusion incohérente des noyaux. La proportionnalité de la double différentielle de la section efficace incohérente à un phonon vis à vis de la densité d'états exprimée dans l'équation 35 dérive de l'équation 33 si la moyenne

$$
\left.\left\{\sum_{j} \sum_{k=1}^{N} \mid \vec{Q} \cdot \vec{e}_{j}(k)\right\}^{2}\right\}_{w_{j}-w}
$$

faite sur tous les modes de fréquences $\omega_{j}=\omega$ est indépendante de $\omega$. C'est le cas du système cubique et des systèmes complètement isotropes. On peut montrer par une simple permutation des directions dans l'équation 36 que la moyenne ci-dessus est rigoureusement proportionnelle à $(1 / 3) Q^{2}$ dans le cas de la symétrie cubique ${ }^{19}$. L'équation 35 nous indique que la diffusion incohérente à un phonon a lieu pour tous les $\vec{Q}$ et est isotrope, i.e. est indépendante de la direction de $\vec{Q}$ dans l'espace réciproque ${ }^{20}$.

Pour des diffuseurs cohérents on est obligé d'invoquer l'approximation incohérente $[19,20]$ afin d'extraire $G(\omega)$ du spectre INS. L'approximation incohérente consiste à supposer que

$$
\int_{Q_{\min }}^{Q_{\max }}\left(\frac{\mathrm{d}^{2} \sigma}{\mathrm{d}(\hbar \omega) \mathrm{d} \Omega}\right), Q \mathrm{~d} Q \approx \int_{Q_{\min }}^{Q_{\max }}\left(\frac{\mathrm{d}^{2} \sigma}{\mathrm{d}(\hbar \omega) \mathrm{d} \Omega}\right), Q \mathrm{~d} Q
$$

pour un échantillonage suffisamment large en $Q$, et où l'expression de droite se rapporte à un échantillon incohérent hypothétique. On peut tester la validité de l'approximation incohérente en comparant les valeurs de $G(\omega)$ pour différentes régions d'échantillonage dans l'espace des $\vec{Q}$. Une autre possibilité est de faire l'estimation des erreurs induites par l'approximation incohérente en faisant des simulations sur ordinateur. On obtient déjà de bons résultats avec des modèles dynamiques d'un faible degré de sophistication. En général, tant l'expérience [6] que la simulation [20] montrent que les erreurs excèdent rarement $20 \%$, même dans les cas les plus défavorables. Dans le cas d'un système à multiples composantes, on peut toujours écrire la fonction de diffusion dynamique sous la forme [20]

$$
S(\vec{Q}, \omega)=A(\vec{Q}, \omega) G_{w}(\omega)
$$

avec de façon analogue à l'équation 35

${ }^{19}$ Cette relation a été utilisé lors de l'établissement de l'éq. 35 .

${ }^{20}$ Il est cependant recommandé de se rappeler que dans le cas de systèmes fortement anisotropes, le traitement cidessus et par suite l'équation 35 ne s'appliquent pas rigoureusement. En exemple on peut prendre une structure en feuillet telle que le graphite avec des forces de cohésion faibles dans la direction d'empilement. Dans ce cas, les basses fréquences seront dominées par les modes dont les vecteurs propres $\vec{e}_{j}(k)$ sont parallèles à la direction d'empilement, et ce pour tous les atomes $k$. En conséquence le signal incohérent INS à partir d'un monocristal de ce matériau dépend de la direction de $\vec{Q}$ et la variation en $|\vec{Q}|$ pour une mesure moyennée avec une poudre peut être plus compliquée qu'indiqué dans l'éq. 35 . 


$$
A(\vec{Q}, \omega)=\frac{\hbar\left(\vec{b}^{2} e^{-2 W(Q)}\right)_{a v}}{2 M_{a v}} \frac{Q^{2}}{\omega}[n(\omega)+1]
$$

avec

$$
M_{a v}=\frac{1}{N} \sum_{k} m_{k} \quad \text { and } \quad\left(\vec{b}^{2} e^{-2 W(Q)}\right)_{a v}=\frac{1}{N} \sum_{k} \bar{b}_{k}^{2} \exp \left(-2 \mathrm{~W}_{\mathrm{k}}(Q)\right)
$$

Les facteurs Debye-Waller $W_{k}(Q)$ étant moyennés sur toutes les directions de $\hat{Q}$ comme on l'a exposé précédemment pour le cas à une composante. Ainsi le résultat obtenu à partir des données de la diffusion inélastique via l'équation 38 est parfaitement défini. Cette $G_{w}(\omega)$ est appelée la densité d'états vibrationnels généralisée (DEVG) qui diffère de la densité d'états vibrationnels actuelle (éq. 14) par ce que les contributions des différents types de noyaux $\{k\}$ aux facteurs de forme des modes $\{j\}$ sont moyennées avec leur pouvoir de diffusion $\left\{\sigma_{\mathrm{k}} / M_{\mathrm{k}}\right\}^{21}$. Les facteurs de correction $A(Q, \omega)$ peuvent se déterminer, respectivement pour le cas cohérent et le cas incohérent, à partir des équations 33 et 34 . Dans les cas où le but de l'expérience est de vérifier un modèle dynamique la façon correcte de procéder est de calculer à partir de la théorie la densité d'états généralisée $G_{w}(\omega)$ ou même encore mieux $S(\vec{Q}, \omega)$.

Sans modèle théorique on doit être conscient des erreurs faites en identifiant aveuglément $G_{w}(\omega)$ avec $G(\omega)$. Ces erreurs peuvent être très importantes comme nous pouvons le montrer avec l'exemple simple de la glace. La glace étant un cristal moléculaire typique présente plusieurs bandes d'excitations. Aux basses fréquences ( $\hbar \omega<40 \mathrm{meV}$ ) on trouve les vibrations impliquant une translation du centre de gravité. Négligeons pour le moment le fait que les phases de la glace sont caractérisées par plus d'une molécule par maille primitive. Si on suppose un découplage complet avec les modes de libration discutés ci-dessous, alors, dans cette région des basses fréquences, le système peut être considéré comme atomique (l'atome étant la molécule $\mathrm{H}_{2} \mathrm{O}$ ) et le poids de chacun des modes translationnels dans le facteur de structure dynamique est déterminé par $2 \sigma_{i}(\mathrm{H}) / m\left(\mathrm{H}_{2} \mathrm{O}\right)$, tant en se rappelant que les vecteurs propres $\mathrm{e}_{\mathrm{j}}$ sont normalisés à l'unité et que la diffusion est dominée par la section efficace incohérente de l'hydrogène. La bande suivante d'excitations contient les librations. Comme les translations les librations sont des modes externes, i. e. qu'elles n'impliquent pas de distorsions des molécules $\mathrm{H}_{2} \mathrm{O}^{22}$. Pour des librations idéales le centre de gravité est au repos (voir le découplage mentionné ci-dessus). Comme le centre de gravité est proche de l'atome d'oxygène, c'est avec une bonne approximation qu'on peut considérer que les modes normaux n'ont des composantes non nulles que seulement pour les atomes d'hydrogènes. Le poids de chaque mode librationnel dans le facteur de structure dynamique est par suite déterminé par $\sigma_{i}(\mathrm{H}) / m(\mathrm{H})$. Ceci signifie que, bien que les deux bandes contiennent le même nombre de modes, l'intensité de la bande librationnelle sera multipliée par un facteur proche de 9 par rapport à une bande translationnelle hypothétique qui aurait la même fréquence. Si on ignore ce fait et on interprète simplement la densité d'état généralisée comme étant la vraie densité d'états, alors cela

\footnotetext{
${ }^{21}$ D'un point de vue strict - et comme on peut aisément le vérifier à partir de l'éq. 38 combinée aux éq. 33 et 34 - la pondération concerne aussi les facteurs Debye-Waller. Nous négligerons cette complication supplémentaire par la suite.

${ }^{22}$ Les modes internes de la molécule $\mathrm{H}_{2} \mathrm{O}$ se trouvent à des fréquences encore plus élevées.
} 
amène d'énormes erreurs dans ce cas éminemment défavorable. Ceci se confirme grâce à des calculs plus élaborés impliquant des simulations de dynamique moléculaire [21].

Nous devons maintenant discuter les contributions multi-phonons à $S(\vec{Q}, \omega)$. Comme on l'a déjà mentionné, ceux-ci croissent fortement à la fois avec $T$ et avec $\mathrm{Q}$. Par suite on peut les négliger bien souvent dans les expériences à basse température impliquant des neutrons froids. Cependant, dans d'autres cas ils doivent être déterminés jusqu'à un ordre suffisamment élevé. Dans le cas d'un système isotrope ou cubique à une composante atomique, la section efficace inélastique incohérente, généralisée à des termes de phonons d'ordres plus élevés, peut s'exprimer sous la forme d'une série ${ }^{23}[22,23,21]$

$$
\left(\frac{d^{2} \sigma}{d(\hbar \omega) d \Omega}\right)_{\mathrm{inc}}=\frac{N \sigma_{i}}{4 \pi} \frac{k_{f}}{k_{i}} e^{-\frac{h \omega}{2 k_{b} T}} e^{-r Q^{2}} \sum_{n=1}^{\infty}\left(\frac{\hbar^{2} Q^{2}}{2 m}\right)^{n} \frac{T_{n}(\hbar \omega)}{n !}
$$

où nous avons employé la forme symétrisée de la fonction de diffusion, i. e. la fonction de diffusion multipliée par $\exp \left(-\hbar \omega / 2 k_{b} T\right)$. En utilisant l'éq. 16 on peut exprimer le déplacement carré moyen ici indiqué comme $\gamma$ comme étant

$$
\gamma \equiv \overline{u^{2}}=\frac{\hbar^{2}}{2 m} \int_{0}^{\infty} \frac{G(\hbar \omega)}{\hbar \omega} \cosh \left(\frac{\hbar \omega}{2 k_{b} T}\right) d(\hbar \omega)
$$

Les fonctions $T_{n}(\hbar \omega)$ dans le développement multi-phonon de l'éq. 41 sont définies de façon récursive grâce à l'expression

$$
T_{n}(\hbar \omega)=\int_{-\infty}^{\infty} T_{1}\left(\hbar \omega-\hbar \omega^{\prime}\right) T_{n-1}\left(\hbar \omega^{\prime}\right) d\left(\hbar \omega^{\prime}\right)
$$

Avec le terme à un phonon

$$
T_{1}(\hbar \omega)=\frac{G(\hbar \omega)}{2 \hbar \omega \sinh \left(\frac{\hbar \omega}{2 \mathrm{k}_{\mathrm{b}} T}\right)}
$$

Par suite, il est possible de calculer la totalité de la diffusion inélastique jusqu'aux ordres de phonons les plus élevés une fois que la densité d'états $G(\omega)$ est connue $e^{24} . G(\omega)$ est déterminée de manière itérative. On calcule d'abord $G(\omega)$ à partir des données comme s'il n'existait pas de contributions d'ordres supérieurs. On porte la première estimation de $G(\omega)$ dans les éq. 42 et 44 afin de déterminer avec l'aide des éq. 43 et 41 la valeur attendue pour la section efficace double différentielle en allant jusqu'à un nombre maximum de phonons $n_{\max }$, qui est librement choisi. L'écart entre cette section efficace calculée et la section efficace actuellement mesurée est utilisé pour affiner l'estimation de $G(\omega)$. Cette procédure est continuée jusqu'à l'obtention de la convergence amenant à $G(\omega)$ d'une façon auto-cohérente.

\footnotetext{
${ }^{23}$ Notez bien que $G(h \omega)=G(\omega) / h$.

${ }^{24}$ Naturellement on doit aussi appliquer l'approximation incohérente aux termes de phonons d'ordre les plus élevés. Dans la formulation exacte ces termes sont très compliqués
} 
Les équations ci-dessus sont valables pour une valeur particulière de $Q$. Si l'on veut appliquer l'approximation incohèrente ou si on doit sommer sur divers bancs de détecteurs pour une meilleure statistique, alors on souhaitera déterminer $G(\omega)$ à partir de données intégrées en $Q$. Cette intégration de l'éq. 41 mène à [22]

$$
\begin{aligned}
\left(\frac{d \sigma}{d(\hbar \omega)}\right) & =\int_{\theta_{\min }}^{\theta_{\max }}\left(\frac{d^{2} \sigma}{d(\hbar \omega) d \Omega}\right)_{\mathrm{inc}} \sin \theta l \theta \\
& =\int_{Q_{\operatorname{man}(\omega)}}^{Q_{\max (\omega)}}\left(\frac{d^{2} \sigma}{d(\hbar \omega) d \Omega}\right)_{\mathrm{inc}} \frac{1}{k_{i} k} Q d Q \\
& =\frac{N \sigma_{i}}{8 \pi} \frac{1}{k_{i}^{2}} e^{-\frac{\hbar \omega}{2 k_{b} T}} \sum_{n=1}^{\infty}\left(\frac{\hbar^{2}}{2 m}\right)^{n} \frac{1}{\gamma^{n+1}}\left(I_{n}\left(\gamma Q_{\max }^{2}\right)-I_{n}\left(\gamma Q_{\min }^{2}\right)\right) \frac{T_{n}(\hbar \omega)}{n !}
\end{aligned}
$$

$\operatorname{avec}^{25}$

$$
I_{n}(x)=\int_{0}^{x} y^{n} e^{-y} d y
$$

Pour des déplacements carrés moyens qui sont petits comparés à $Q_{\min }^{-2}, Q_{\max }^{-2}$ on obtient pour le terme à un phonon

$$
\left(\frac{d \sigma}{d(\hbar \omega)}\right)_{n-1}=\frac{N \hbar^{2} \sigma}{8 \pi m k_{i}^{2}} n(\omega) \frac{G(\omega)}{\omega}\left(Q_{\max }^{4}(\omega)-Q_{\min }^{4}(\omega)\right)
$$

c'est l'équation de départ pour une analyse en termes de densité d'états, de données de temps de vol moyennées en angles. Dans les systèmes à composants multiples la composante multi-phonons est plus impliquée à cause des problèmes évoqués ci-dessus dans le contexte de la densité d'états généralisée.

\section{Intensité de la diffusion inélastique}

Bien que les expressions ci-dessus donnent l'intensité de la diffusion en valeur absolue, il est intéressant de comparer la diffusion inélastique au signal élastique. Nous allons le faire pour un système simple à un seul composant qui est un diffuseur totalement incohérent, dans un réseau de symétrie cubique tel que le vanadium. La raison étant que pour un tel système les résultats dérivés dans la section $3.2 \mathrm{~s}$ 'appliquent rigoureusement.

Dans ce cas la diffusion élastique est isotrope et donnée simplement par

${ }^{25} \Theta_{\min }$ et $\Theta_{\max }$ se réfèrent aux maximum et minimum des angles de diffusion pour l'intégration dans une géométrie de spectromètre à temps de vol tel que IN6. $Q_{\min }$ et $Q_{\max }$ dépendent de la fréquence à cause de la dispersion parabolique des neutrons. 


$$
\left(\frac{d \sigma}{d \omega}\right)_{\mathrm{inc}}^{\mathrm{e} 1}=N \sigma_{i} e^{-2 \omega(Q)}
$$

Pour le cas élastique on peut utiliser l'éq. 35. Si on est intéressé uniquement par les plus basses fréquences alors on peut exploiter le fait que ce sont des modes acoustiques, i.e. qu'elles présentent la dispersion:

$$
\omega(\vec{q})=|\vec{q}| \cdot c_{j, \hat{q}}
$$

où $c_{j, \hat{q}}$ représente la vitesse du son suivant la direction $\hat{q}$ pour la branche acoustique $j$. La densité

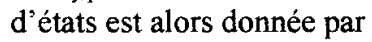

$$
G(\omega)=\left(\frac{V}{N}\right)\left(\frac{2 \pi^{2}}{c^{3}}\right) \omega^{2}=\frac{3 \omega^{2}}{\omega_{b}^{3}}
$$

où $\mathrm{c}$ représente la vitesse du son moyennée sur les directions et sur les branches. $\omega_{D}$ tel qu'il est défini à partir de l'éq. 50 est la fréquence de Debye du matériau. V est le volume occupé par les $N$ atomes. Utilisant le fait que $n(\omega) \rightarrow k_{b} T / \hbar \omega$ pour $\hbar \omega \rightarrow 0$ on obtient la section efficace inélastique indépendante de $\omega$ en gain d'énergie

$$
\left(\frac{d^{2} \sigma}{d E_{f} d \Omega}\right)_{\mathrm{inc}}=\frac{3 N \sigma_{i}}{8 \pi m} \frac{k_{f}}{k_{i}} Q^{2} e^{-2 W(\bar{Q})}\left(\frac{k_{b} T}{\hbar \omega_{D}^{3}}\right)
$$

Ceci donne le rapport des signaux élastique et inélastique

$$
\left(\frac{d \sigma}{d \Omega}\right)_{\mathrm{inc}}^{\mathrm{el}} / \int_{0}^{\hbar \omega_{l}}\left(\frac{d^{2} \sigma}{d(\hbar \omega) d \Omega}\right)_{\mathrm{inc}}^{\text {inel }} d \hbar \omega \approx \frac{4 \pi}{3} \frac{2 m}{\hbar^{2} Q^{2}}\left(\frac{\left(\hbar \omega_{D}\right)^{3}}{\left(\hbar \omega_{l}\right)\left(k_{b} T\right)}\right)
$$

où on a employé $k_{i} \approx k_{f}$ pour les faibles transferts d'énergie. Par suite, le rapport est définie par l'énergie caractéristique $\hbar \omega_{D}$ du spectre de vibration, l'énergie thermique $k_{b} T$ et l'intervalle d'intégration $\hbar \omega_{k}$. Ces valeurs sont toutes dans la gamme entre zéro et $100 \mathrm{meV}$. Les énergies de Debye typiques sont de l'ordre de $40 \mathrm{meV}$, alors que $k_{b} T$ à température ambiante est environ 30 $\mathrm{meV}$. En prenant $\mathrm{Q}=2 \AA^{-1}$ et une masse atomique de $50 \mathrm{uma}$, on obtient pour $\hbar^{2} Q^{2} / m$ environ 0.3 $\mathrm{meV}$. Cette estimation simple donne pour les intensités inélastiques une gamme de 0 à $10 \mathrm{meV}$ qui est de 100 à 1000 fois plus petite que ce qu'on recueille dans le canal élastique. Ceci explique pourquoi il est si important de maintenir le bruit de de fond à un faible niveau lors des expériences inélastiques. Notez aussi que le facteur Debye-Waller qui affecte également les intensités élastiques et inélastiques n'entre pas en ligne de compte dans ce rapport. 


\subsection{Relations de dispersion}

Etant donné que le facteur de structure dynamique exprimé dans les éq. 33 et 34 est valable de façon générale pour tous système d'atomes il doit également s'appliquer au cas particulier des cristaux. Cependant, il ne démontre pas explicitement les conséquences de l'invariance par translation pour le spectre de diffusion inélastique des neutrons. Pour remédier à ce défaut nous utiliserons la classification des déplacements atomiques en termes d'ondes planes comme c'est fait dans l'éq. 19. La somme sur tous les atomes du système dans l'éq. 29 se décompose alors en une somme sur les atomes dans la maille primitive et ensuite en une somme sur l'espace réciproque. Après une normalisation appropriée on obtient pour le facteur de structure dynamique

$$
\begin{gathered}
S(\vec{Q}, \omega)=\frac{(2 \pi)^{3}}{v} \sum_{G} \sum_{q, j} \frac{\left|F_{j}(\vec{Q})\right|^{2}}{\omega_{j}(\vec{q})}\left[\left(n_{j}(\vec{q})+1\right) \delta\left(\omega-\omega_{j}(\vec{q})\right) \delta(\vec{Q}-\vec{G}+\vec{q})+\right. \\
\left.n_{j}(\vec{q}) \delta\left(\omega+\omega_{j}(\vec{q})\right) \delta(\vec{Q}-\vec{G}-\vec{q})\right]
\end{gathered}
$$

$v$ est le volume de la cellule primitive. $\vec{G}$ sont les vecteurs des points du réseau réciproque et $\vec{q}$ est dans la première zone de Brillouin. Le facteur de structure correspondant aux vibrations $j$ se simplifie dans le cas d'un cristal en

$$
F_{j}(\vec{Q})=\sum_{k=1}^{r} \frac{\left|\vec{b}_{k}\right|}{\sqrt{m_{k}}} e^{-W_{k}(\vec{Q})}\left[\vec{Q} \cdot \vec{e}_{j}(\vec{q})\right] e^{-i \bar{Q}_{k} \vec{d}_{k}}
$$

Le vecteur $\vec{d}_{k}$ donne la position de l'atome dans la cellule primitive. La présence des fonctions $\delta$ dans l'éq. 53 a pour conséquence que la diffusion pour un transfert de moment donné $\vec{Q}=\vec{k}_{i}-\vec{k}_{f}$ se produit idéalement uniquement pour des transferts d'énergie $\left|E_{f}-E_{i}\right|=\hbar \omega_{j}, j=1, \ldots 3 r$. Dans le cas où l'approximation harmonique n'est pas valable les signaux acquièrent une largeur intrinsèque qui est directement reliée à la durée de vie de l'excitation.

La classification suivant $\vec{q}$ doit prendre en compte les règles de sélection de la symétrie de translation du cristal. Suivant quelques directions de haute symétrie dans l'espace réciproque $F_{j}(Q)$ peut être rigoureusement nul pour des sous-ensembles de branches de dispersion $\{j\}$. Comme ces règles de sélection dépendent uniquement de la symétrie elles peuvent être déterminées rigoureusement indépendamment de la dynamique du système, i. e. sans aucune connaissance des potentiels interatomiques [10].

\section{Expériences de diffusion inélastiques des neutrons}

Quel instrument utiliser? Il existe un très large assortiment d'appareils permettant de faire des expériences de diffusion inélastique, tant pour les sources continues que pour les sources pulsées. Ce n'est pas notre but de les comparer en détail ici. Les spectromètres trois-axes sur les sources continues offrent un flux incident très élevé et fonctionnent en mode pas à pas, i. e. $S(\vec{Q}, \omega)$ est déterminé pour un point $(\vec{Q}, \omega)$ à la fois. Ils sont l'idéal pour la détermination des relations de dispersion dans les monocristaux. Ceci est particulièrement valable quand la recherche peut être 
restreinte à une région $(\vec{Q}, \omega)$ particulière. Les spectromètres trois-axes sont en concurrence avec une nouvelle génération de spectromètres à temps de vol adaptés à des expériences avec des monocristaux (par ex. MAPS près de la source de neutron par spallation ISIS). Ils ont déjà montré leur grand potentiel dans le cas de recherches sur des systèmes à basse dimensionnalité. Dans tous les cas le temps de vol est la méthode de choix pour la mesure des densités d'états car elle fournit simultanément des informations pour une grande partie de l'espace $(\vec{Q}, \omega)$. Cette large bande d'information est essentielle pour les divers procédés d'établissement de moyennes discutés dans la section 3.2. La perte d'intensité due à la nécessité de pulser un faisceau continu de neutrons dans le cas des instruments à temps de vol basés près des sources à réacteurs est souvent compensée par la plus grande flexibilité en terme de résolution et de répétition. Aussi même les spectromètres à temps de vol ont encore un avenir auprès des réacteurs et peuvent rivaliser avec les spectromètres existant près des sources pulsées.

Pour la spectroscopie vibrationnelle, en particulier avec des échantillons cohérents, il est bien sûr souhaitable d'avoir accès à la gamme de $\vec{Q}$ la plus large possible. Cependant, cela est seulement possible en ayant de grandes énergies incidentes ce qui dans la plupart des cas signifie une sévère perte dans la résolution en énergie. Quelle résolution en énergie est nécessaire dépend du problème étudié. Par suite le choix de l'instrument et en particulier des paramètres instrumentaux tels que les longueurs d'onde incidentes etc. doit être examiné cas par cas.

Comment faire le traitement des données? Une fois l'expérience réalisée, les données brutes doivent être converties en variables physiques utilisables. Dans le cas de mesures avec des monocristaux ceci peut s'avérer une tâche plutôt facile, si les pics de phonons sont nettement séparés. En principe, il pourra être possible de cartographier les relations de dispersion complètes simplement en identifiant les pics dans le facteur de structure dynamique. En pratique la résolution expérimentale à la fois en $\omega$ et en $\vec{Q}$ gâche le jeu ${ }^{26}$. Dans la plupart des cas la résolution en énergie est plus mauvaise que l'espacement des feuillets de dispersion. Par suite, au lieu d'un ensemble de pics on mesure des structures compliquées. Pour retrouver l'information sur les fréquences du réseau, il est indispensable dans la plupart des cas de faire des calculs avec des modèles dynamiques combinés avec une simulation de la résolution. Les calculs permettent de prédire les intensités diffusées en fonction de $Q$ et par suite guident la recherche expérimentale de branches de dispersion spécifiques avec l'aide de l'éq. 53.

Dans le cas d'échantillons polycristallins ou amorphes la diffusion est continue dans l'espace $(\vec{Q}, \omega)$. En conséquence, on ne doit pas s'attendre à un spectre fortement structuré. Par suite, la détermination des intensités est essentielle car elle contient l'information concrète. Ceci implique une suite indispensable de mesures de bruit de fond et de normalisation. Le problème du bruit de fond devient particulièrement important quand on utilise des environnements lourds des échantillons tels que des cellules à pression [27]. En dehors de ces sources externes de perturbation

\footnotetext{
${ }^{26}$ Dans les expériences de diffusion inélastique des neutrons on porte souvent une attention limité à la résolution en $Q$ de l'appareil. L'idée sous-jacente est que le gradient de la surface de dispersion aussi bien que le facteur de structure associé change très peu dans le volume limité par l'ellipsoìde de résolution. Cependant, cette hypothèse est seulement justifiée à la condition que la taille de l'ellipsoïde soit vraiment petite à l'échelle à laquelle les fréquences propres et les vecteurs propres changent de façon appréciable. C'est rarement le cas. Typiquement, cette dernière échelle est donnée par la taille de la zone de Brillouin.
} 
les données sont intrinsèquement perturbées par la diffusion multiple et par l'absorption. On ne peut jamais éviter complètement la diffusion multiple. Afin d'avoir une statistique raisonnable pour les données il est nécessaire d'avoir une certaine quantité de matière dans le faisceau. Plus il y a de matériau plus est grande la probabilité qu'un neutron soit diffusé plus qu'une fois. Comme la diffusion inélastique est très faible (voir la discussion de la section 3.3) la diffusion multiple impliquant plus d'un événement inélastique peut être négligée. Ceci implique que la diffusion multiple mélange différentes valeurs de $Q$ mais pas différentes énergies, i. e. la diffusion multiple est locale en $\omega$ mais non locale en $Q$. Ce mélange par diffusion multiple est plus ou moins isotrope. Comme le signal du à un phonon décroît vers zéro comme $Q^{2}$ (voir par ex. l'éq. 35) cette contribution constante de la diffusion multiple dominera le signal aux faibles $Q$. Si on ne le prend pas en compte convenablement il provoquera des erreurs importantes dans l'interprétation des données. Pour des valeurs plus grandes de $Q$ l'influence de la diffusion multiple est moins importante et peut souvent être ignorée sans crainte. Comme le pourcentage de diffusion multiple dépend de la région en $(\vec{Q}, \omega)$ qui est explorée par les neutrons incidents ou sortants diffusés élastiquement, elle dépend des paramètres expérimentaux en violation avec le découplage entre le neutron sonde et la fonction de diffusion exprimée dans l'éq. 23. On peut trouver des discussions plus détaillées sur la diffusion multiple dans les références [21] et [24].

\section{Exemple : Spectre des modes externes des fullérènes}

Dans cette section nous donnons un exemple explicite de l'application de la diffusion inélastique des neutrons à la science des matériaux qui illustre les sujets développés précédemment. Nous avons choisi l'étude des vibrations externes des fullérènes parceque ce sujet nous permet de montrer comment on peut obtenir de précieuses informations concernant les topologies des liaisons, les forces de liaison et certains mécanismes de réactions chimiques

Il y a une dizaine d'années on a observé expérimentalement, pour la première fois, des molécules entièrement en carbone et complètement fermées (fullérènes), cette découverte a été récompensée par un prix Nobel de chimie en 1996. Quelques années après la première identification de fullérènes isolés on a découvert un procédé de fabrication qui fournissait des quantités macroscopiques de $\mathrm{C}_{60}$ et de $\mathrm{C}_{70}$, les deux membres les plus stables de la famille des fullérènes. Ceci a ouvert une opportunité pour l'étude de leurs propriétés à l'état solide [29] où la diffusion des neutrons tant élastique que inélastique (INS) a joué un rôle vital dès le début.

A haute température, les fullérènes forment des structures cristallines où les molécules sont en rotation presque libre (phase rotationnelle), avant de s'évaporer dans la phase gazeuse. Dans une représentation de chimie conventionnelle les molécules neutres de fullérène devraient avoir très peu tendance à former des liaisons covalentes entre elles étant donné que toutes leurs liaisons sont en résonance entre elles (molécules aromatiques). Mais on peut tirer la conclusion inverse si on représente la cage comme un ensemble altéré de simples et doubles liaisons. On considère actuellement que les fullérènes se situent entre ces deux extrêmes. Plus tard on a découvert que les fullérènes ont une forte tendance à subir des réactions chimiques pourvu qu'elles soient catalysées par des conditions convenables telles que - l'exposition à la lumière [30], de fortes pressions à température élevée [31] ou un dopage avec une quantité convenable d'ions alcalins (i. e. $\mathrm{AC}_{60}, \mathrm{~A}=$ $\mathrm{K}, \mathrm{Rb}, \mathrm{Cs}$ [32] ou $\mathrm{Na}_{4} \mathrm{C}_{60}$ [33])- en formant une grande variété de topologies de polymères. Les 
systèmes $\mathrm{AC}_{60}$ et $\mathrm{Na}_{4} \mathrm{C}_{60}$ sont particulièrement intéressants car leur polymérisation est réversible et peut être étudiée en fonction de la température.

Comment peut-on identifier des liaisons intercages grâce à la diffusion inélastique des neutrons?

Dans la figure 4 nous avions déjà présenté $G(\omega$,$) la densité d'états de phonons, pour quelques$ systèmes de fullérènes typiques. Le monomère $\mathrm{C}_{60}$ qui est là dans la phase plastique, présente un intervalle bien développé entre 6 et $30 \mathrm{meV}$ qui sépare les modes de vibrations internes des modes externes de translation ${ }^{27}$. Le premier mode interne est vu à environ $30 \mathrm{meV}$ et est élargi par la rotation moléculaire. Le 'gap' est un signe direct de la séparation des échelles d'énergies qui déterminent d'une part la cohésion d'une cage isolée et de l'autre la cohésion d'un ensemble de cages. La liaison inter-cages influence les spectres des cristaux moléculaires essentiellement de deux façons. D'un coté la déformation des cages provoque des changements de fréquence des modes internes. Leur interprétation exige des calculs théoriques très évolués. D'un autre coté le système subit une rupture plus ou moins prononcée de son caractère de cristal moléculaire, aboutissant à une variation de densité spectrale dans la région du 'gap' (0-30 meV) du composé monomère. L'analyse de ces modes de gap est quasiment directe tant qu'il n'y a pas de fort mélange entre les degrés de liberté internes et externes. Bien que les modes de 'gap' correspondent à des excitions collectives leurs fréquences sont déterminées principalement par l'environnement immédiat des molécules. En conséquence, on peut obtenir relativement aisément des informations sur le type de liaison et la force de liaison à partir des données spectroscopiques même en présence de désordre à longue distance tels que des défauts d'empilement [34].

Comme il n'existe pas de gros monocristaux de fullérènes polymérisés purs ou dopés les expériences d' INS doivent s'effectuer avec des poudres. Les spectres présentés ici ont été obtenus sur le spectromètre à temps de vol et neutrons froids IN6 de l'ILL qui combine une bonne résolution dans la gamme d'énergie d'intérêt majeur (0-30 meV) avec une forte intensité. Les échantillons ont une transmission de 90 à $95 \%$. Comme nous ne sommes pas intéressés par les informations à faibles $Q$, dans la plupart des cas appliquer l'approximation de diffusion incohérente multiple n'est pas un problème. Le carbone est un diffuseur entièrement cohérent. On peut tester la validité de l'approximation incohérente en comparant les $G_{w}(\omega)$ obtenus en sélectionnant différentes régions de l'espace des $Q$. On trouve qu'aussi longtemps que $Q_{\max }(\omega)$ est supérieur à 3.0 $\AA^{-1}$ et que le volume échantillonné dans l'espace réciproque est suffisamment grand dépend seulement légèrement de la gamme de $Q$ explorée ( à part des changements qui peuvent résulter de différences dans la résolution en énergie). Ainsi, dans le cas de l'instrument IN6 employant des neutrons d'énergie $E_{i}=4.75 \mathrm{meV}$ cette condition est satisfaite pour $\hbar \omega$ supérieur à quelques meV. Des tests sont nécessaires du fait de la forme plutôt particulière de la molécule $\mathrm{C}_{60}$, qui ressemble à une sphère creuse. Cette aspect fait que le facteur de forme dynamique [35] pour les modes de libration (molécule de $\mathrm{C}_{60}$ librant autour de son centre de masse) est pratiquement nul pour des valeurs de $Q$ plus petites que $2.5 \AA^{-1}$. Par suite il est évident que l'emploi d'une gamme trop restreinte de $\mathrm{Q}$ quand on emploie l'approximation incohérente va exclure les excitations de libration. Cet exemple illustre le soin qu'il faut prendre quand on utilise l'approximation incohérente.

Les données sont normalisées à un standard de vanadium et corrigées du bruit de fond avant d'être converties en susceptibilités généralisées ou en densités d'états. Pour les systèmes dopés cette densité d'états est une fonction généralisée $G_{w}{ }^{\exp }(\omega)$ en appliquant l'approximation incohérente. On calcule et on corrige les coefficients multi-phonons et Debye-Waller de la manière auto-cohérente ${ }^{27}$ Les réorientations rotationnelles dans la phase plastique provoquent de la diffusion quasi-élastique et par suite ne
sont pas bien décrites par $G(\omega)$ 
exposée en section 3.2. Afin de les comparer avec l'experience on calcule a partir des modèles les densités d'états généralisées $G_{w}{ }^{\text {theary }}(\omega)$.

Dans les systèmes en cause ici, toutes les parties de $G(\omega)$ réagissent à des liaisons inter-cage. Le spectre du dimère présente des pics bien nets autour de $10 \mathrm{meV}$ qui sont dus aux modes internes des haltères de $\left(\mathrm{C}_{60}\right)_{2}$ (voir la fig.4). Comme les 'haltères' interagissent au moyen de faibles forces de Van der Waals, elles sont dynamiquement isolées les unes des autres. Ceci entraîne des modes d'Einstein plats [12] qui provoquent des structures avec des pics dans $G(\omega)$ (fig.5). Déjà qualitativement le spectre de la phase polymère diffère de celui de la phase dimère (fig. 4). Par suite de la connectivité infinie du réseau suivant les directions de polymérisation, les branches de phonons externes montrent de la dispersion comme dans le cas du monomère (fig.1) ce qui provoque des caractéristiques élargies dans $G(\omega)$. Il est évident que le réseau à connexions 3D du système polymère, à part des changements relativement faibles dans la matrice d'interaction, peut être considéré comme semblable au système monomère, $i$. e. les spectres des modes externes sont reliés par une remise à l'échelle de l'axe des fréquences. Comme il faut conserver le poids des modes externes il est possible de déduire la dimensionnalité du réseau à partir d'un simple examen des intensités dans les régions du gap et des interactions Van der Waals. Si on les compare à l'intensité du premier mode interne, les intensités au dessous de $6 \mathrm{meV}$ diminuent tandis que celles au dessus de $6 \mathrm{meV}$ croissent lorsqu'on passe des chaînes polymères $1 \mathrm{D}$ aux feuillets polymères 2D (fig.4).

Pour extraire une information quantitative des vibrations de basses fréquences un calcul de dynamique de réseau est indispensable. Dans ce but nous avons employé un modèle hybride combinant des champs de force et des interactions atome-atome dérivées d'un potentiel [6]. Dans ces calculs tous les atomes de carbone sont pris comme des unités dynamiques indépendantes. Les interactions $\mathrm{C}-\mathrm{C}$ dans les boules sont décrites par un champ de force consistant en des forces longitudinales et fonction des angles de liaison, de Born-von Kármán (voir la section 2.2). On introduit des potentiels atome-atome de Van der Waals pour les interactions $\mathrm{C}-\mathrm{A}(\mathrm{A}=\mathrm{Na}, \mathrm{K}, \mathrm{Rb}$, $\mathrm{Cs})$ ainsi que pour les liaisons entre deux atomes entre deux atomes de carbone appartenant à deux molécules différentes. La mauvaise connaissance des potentiels $\mathrm{C}$-A dans les fullérènes constitue le problème principal pour les calculs. Nous avons employé un ensemble minimum de 3 paramètres pour décrire les liaisons intercages. Ils consistent en des constantes de force d'étirement et déformation des angles. Dans la plupart de ces cas la valeur de ces paramètres peut se transférer à partir des interactions intra boule ce qui laisse peu de besoin d'ajustement.

Afin de comparer avec les données expérimentales la fonction théorique $G_{w}^{\text {theory }}(\omega)$ est convoluée avec la fonction de résolution de l'instrument.

Les modèles présentés ci-dessus donnent une description très satisfaisante de $G(\omega)$ pour tous les réseaux de fullérènes étudiés. Nous donnerons trois exemples bien représentatifs : (i) un système polymère à chaînes $1 \mathrm{D}\left(\mathrm{RbC}_{60}\right)$ (ii) un système dimère $\left(\mathrm{RbC}_{60}\right)$ (iii) un système polymère à feuillets $2 \mathrm{D}\left(\mathrm{Na}_{4} \mathrm{C}_{60}\right)$.

Sur la fig. 1 nous présentons les courbes de dispersion de phonons calculées ainsi que les densités d'états pour la chaîne de polymère $\mathrm{RbC}_{60}$. La liaison via 4 atomes de contact à partir d'un pont cyclo-butane (liaison $|2+2|$ ) qui avait été proposée sur la base de recherches structurales est confirmée par les données spectroscopiques. Les forces de rappel dans l'unité cyclo-butane $\mathrm{C}_{4}$ peuvent se déduire directement à partir d'interactions équivalentes sur la cage confirmant le caractère fortement covalent de ces liaisons. Les forces de rappel angulaires pour les librations autour d'axes perpendiculaires à la direction de la chaîne sont très importantes, ce qui aboutit à des fréquences de libration au dessus de la bande d'étirement de la chaîne. D'un autre coté les chaînes sont très rigides si on considère leur torsion. 

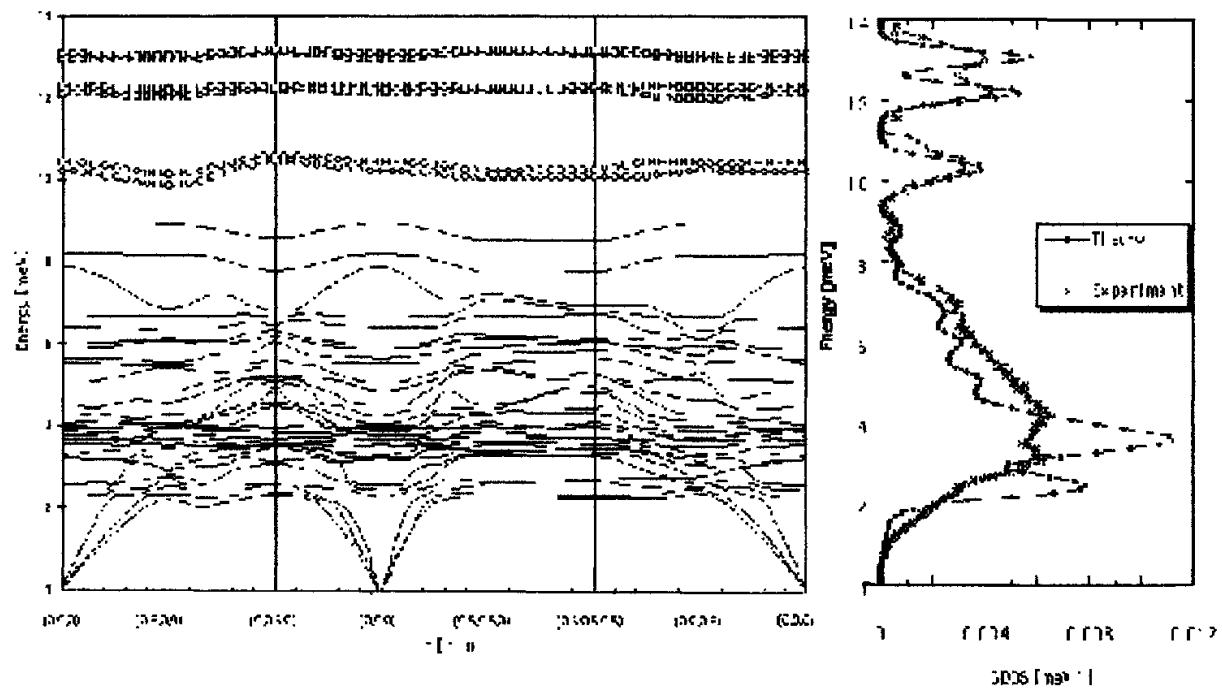

Figure 5 : Relations de dispersion pour la phase dimère $\mathrm{RbC}_{60}$. Il y a quatre unites $\mathrm{RbC}_{60}$ dans la maille primitive. Les dimères sont alignés suivant $x$. Les branches optiques sont plates ce qui entrâne des pics étroits dans la densité d'états. Les branches de plus hautes fréquences correspondent aux librations autour d'axes perpendiculaires aux axes longs des 'haltères' (carrés). On trouve les modes d'étirement des haltères dans la région des $10 \mathrm{meV}$ (cercles).

La modélisation de la dynamique des dimères de $\left(\mathrm{C}_{60}\right)_{2}$ s'oriente vers une liaison impliquant un contact entre deux atomes seulement. Une liaison $|2+2|$ comme dans le polymère exigerait des constantes de force trop basses d'un point de vue physique [6]. Les courbes de dispersion du dimère présentent des bandes plates autour de $10 \mathrm{meV}$ qui sont dues aux modes internes des 'haltères' (fig. 5). Comme les 'haltères' interagissent seulement à travers de faibles forces de Van der Waals elles sont dynamiquement isolées les unes des autres ce qui explique le caractère Einstein de ces modes. On attribue les bandes de fréquences les plus élevées aux librations des cages autour d'axes perpendiculaires aux 'haltères'. La bande de plus basse fréquence correspond au mode d'étirement. La torsion de 'l'haltère' autour de son axe principal met seulement en jeu de faibles forces de rappel et est cachée dans la bande de basse fréquence. Le spectre acoustique est significativement plus mou que dans le polymère comme on doit s'y attendre pour un système moléculaire d'haltères $\left(\left(\mathrm{C}_{60}\right)_{2}^{-2}\right)$ seulement liées par des forces de Van der Waals. Ce sont les liaisons les plus faibles dans une chaîne qui déterminent sa rigidité aux grandes longueurs d'ondes. 


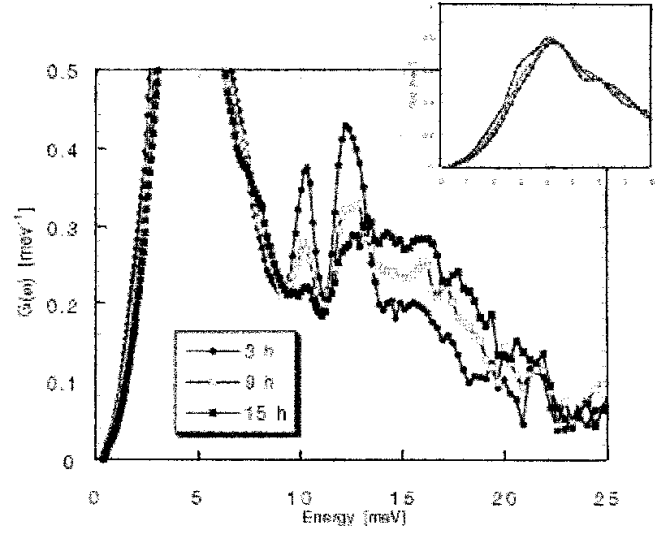

Figure 6: Evolution de $G(\omega)$ pour $\mathrm{RbC}_{60}$ à $260 \mathrm{~K}$ pendant 15 heures à partir du démarrage de la phase dimère. Chaque courbe correspond à des données accumulées pendant 3 heures. Tandis que le pic dimère disparaît la distribution élargie du polymère croit. L'encart montre en fonction du temps, le déplacement vers les hautes fréquences des modes acoustiques et du mode libron.

Etudes de réactions chimiques avec la diffusion inélastique des neutrons. Des systèmes de fullérènes liés offrent de remarquables possibilités d'étudier la formation de liaisons chimiques dans un environnement cristallin. La plupart des phases polymères de composés purs sont métastables à pression ambiante et se transforment en la phase monomère par chauffage. Dans les composés dopés les phases polymères ( $1 \mathrm{D}$ dans $\mathrm{AC}_{60}$ et $2 \mathrm{D}$ dans $\mathrm{Na}_{4} \mathrm{C}_{60}$ ) sont stables et peuvent se former de façon réversible lors de cycles thermiques. Nous allons donner ici quelques exemples de ces transitions qui démontrent l'utilité de l'INS pour leur étude. Des dimères de $\left(\mathrm{C}_{60}\right)_{2}$ se forment quand on trempe du $\mathrm{RbC}_{60}$ depuis la phase plastique haute température jusqu'à la température de l'azote liquide. La phase dimère est métastable et se transforme irréversiblement en la phase polymère avec une vitesse de conversion fortement dépendante de la température. Profitant des signatures nettes laissées dans le spectre inélastique par les modes des 'haltères', on peut suivre ce processus de façon très détaillée par l'INS. La figure 6 montre l'évolution complexe du spectre à $260 \mathrm{~K}$ pendant une période de 15 heures. Le pic dimère perd lentement de l'intensité tandis que croit une distribution large due aux modes des chaînes en développement. Dans la région des basses fréquences le spectre se durcit et les bosses autour de 2 et $3 \mathrm{meV}$ disparaissent. Le raidissement se produit par le fait qu'on passe d'un système d'interactions faibles et fortes alternées à des chaînes homogènes où il y a de fortes interactions. Si on les interprètent comme dues aux modes de torsion basse fréquence des 'haltères' les épaulements autour de 2 et $3 \mathrm{meV}$ doivent aussi se durcir au cours de la polymérisation de la chaîne.

Par suite de considérations de chimie quantique, les dimères liés par de simples liaisons doivent se scinder en monomères avant de se ré-assembler en polymères à liaisons $|2+2|$. Dans le spectre de neutrons il n'y a pas de signes de diffusion quasi-élastique qui indiqueraient une ré-orientation rapide des monomères. Par conséquent, soient les monomères creés par dissociation des dimères sont -sur l'échelle de temps du processus de formation- reliés en unités plus grandes ou bien leur rotation est fortement gênée par rapport aux phases plastiques de $\mathrm{C}_{60}$. Comme il n'est pas possible de décrire le spectre à des étapes intermédiaires par la superposition des fonctions de réponse du polymère pur et du dimère, on peut exclure un transformation via une croissance d'un domaine 
simple. Les données INS, au contraire, indiquent la présence d'une série d'étapes désordonnées intermédiaires, qui peuvent vraisemblablement être composées de chaînes de longueurs finies et qui peuvent être trempées dans un état vitreux.
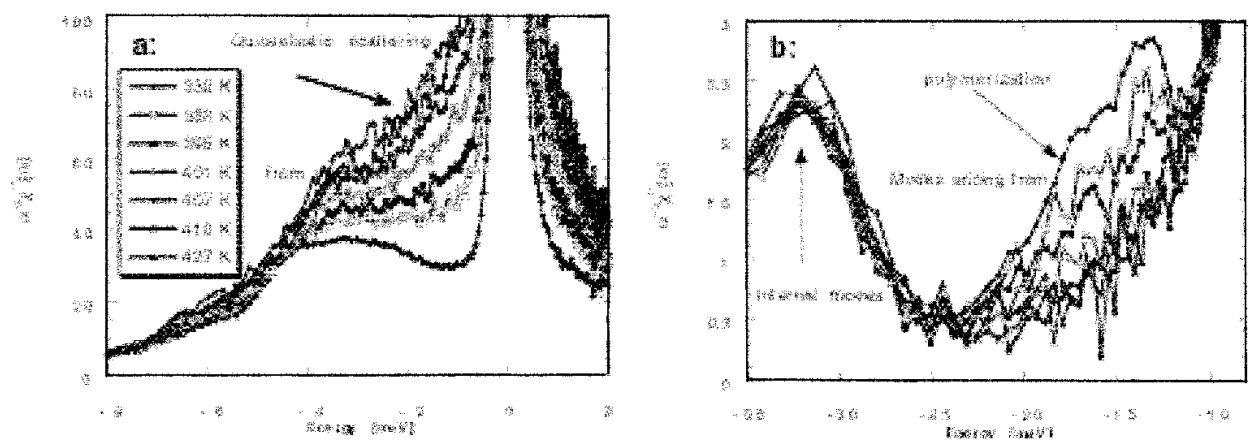

Figure 7: Evolution de $\omega^{-1} \chi^{y}|\omega|$ en fonction de la température en partant d'un polymère relaxé. $\chi^{1}|\omega|$ est la susceptibilité généralisée obtenue avec les données INS intégrées en angles. Pour des énergies faibles en comparaison avec la température $\omega^{-1} \chi^{1}|\omega|$ est proportionnelle à la fonction de diffusion $S(\omega)$. Les étapes intermédiaires peuvent s'exprimer par une superposition linéaire des spectres du polymère pur et du monomère indiquant que la polymérisation induit une séparation de phases (PIPS). Cette séparation est instantanée et stable pour l'échelle de temps des heures jusqu'à $395 \mathrm{~K}$.

La phase polymère de $\mathrm{RbC}_{60}$ est thermodynamiquement stable à température ambiante. Au dessus de $410 \mathrm{~K}$ les polymères doivent complètement se dissocier et les cages de $\mathrm{C}_{60}$, comme mentionné précédemment, exécutent de rapides mouvements de diffusion rotationnelle. En partant d'un échantillon de polymère complètement relaxé, le spectre inélastique montre une évolution en température incompatible avec un système harmonique homogène (fig. 7) à des températures aussi basses que $350 \mathrm{~K}$. Par contraste avec la transition dimère $\Rightarrow$ polymère, la réponse dynamique après la correction de la dépendance harmonique normale en température (susceptibilités) peut s'exprimer comme une superposition de celles obtenues pour le polymère pur et le monomère pur. Cette propriété de superposition est due à la présence de domaines de monomères emprisonnés dans la matrice polymère par suite de la séparation de phases induite par la polymérisation (PIPS). Le pourcentage de monomère croit exponentiellement avec la température. Mais tandis que jusqu'à $395 \mathrm{~K}$ le système est en équilibre thermique (sur une échelle de temps de l'heure), le spectre change visiblement avec le temps au dessus de cette température. Lors du cycle de refroidissement le PIPS peut s'observer aisément en fonction du temps pour des températures inférieures à $380 \mathrm{~K}$. Le montant de signal quasi-élastique du aux monomères diminue graduellement, se saturant quelque temps après à une valeur supérieure à celle obtenue à la même température lors du chauffage du polymère relaxé (hystérésis). L'intensité relative de la diffusion quasi-élastique permet de faire une bonne estimation de la quantité absolue de monomères présents dans le système [6].

Un Grand Merci à Jean Meinnel pour avoir entrepris la traduction du text anglais en français. 


\section{Références}

[1] D. Strauch, A.P. Mayer, and B. Dorner, Z. Physik B 78, 405 (1990)

[2] D. Strauch and B. Dorner, J. Phys.: Condens. Matter 5, 6149 (1993)

[3] H. Schober, D. Strauch, K. Nützel, and B. Dorner, J. Phys.: Condens. Matter 5, 6155 (1993)

[4] H. Schober, D. Strauch, and B. Dorner, Z. Physik B 92, 273 (1993)

[5] R. Mittal, S.L. Chaplot, H. Schober, and T.A. Mary, Phys. Rev. Lett. 86, 4692 (2001)

[6] H. Schober, A. Tölle, B. Renker, R. Heid, and F. Gompf, Phys. Rev. B 56, 5937 (1997)

[7] F. Gompf, W. Reichardt, H. Schober, B. Renker, and M. Buchgeister, Phys. Rev. B 55, 9058 (1997)

[8] D. Strauch, P. Pavone, A.P. Mayer, K. Karch, H. Sterner, A. Schmid, Th. Pletl, R. Bauer, and M. Schmitt, in Festkörperprobleme, Advances in Solid State Physics, Vol. 37, edited by R. Helbig Vieweg, Braunschweig, 1997 , p. 99.

[9] G. Lang, K. Karch, M. Schmitt, P. Pavone, A.P. Mayer, R. K. Wehner and D. Strauch, Phys. Rev. B 59, 6182 (1999)

[10] J.M. Perez-Mato, M. Aroyo, J. Hlinka, M. Quilichini, and R. Currat, Phys. Rev. Lett. 81, 2462 (1998)

[11] Ch. Kittel, Introduction to Solid State Physics, 6th Edition, Wiley (1986)

[12] N.W. Ashcroft and N.D. Mermin, Solid State Physics, Saunders College, Philadelphia (1976)

[13] A.A. Maradudin, E.W. Montroll, G.H. Weiss, and I.P. Ipatova, Theory of Lattice Dynamics in the Harmonic Approximation, Academic, New York, (1971)

[14] C. Cohen-Tannoudji, B. Diu, and F. Laloë, Méchanique Quantique, Tome I et Tome II Hermann Editeurs des Sciences et des Arts, Paris (1977, 1986)

[15] R.J. Bell and P. Dea, Philos. Mag. 25, 1381 (1972)

[16] J.P. Elliot and P.G. Dawber, Symmetry in Physics, Vol. 2, chapter 14, Oxford University Press, New York (1984)

[17] V. F. Sears, Neutron Optics, Oxford University Press, New York (1989)

[18] J. Wuttke, M. Kiebel, E. Bartsch, F. Fujara, W. Petry, and H. Sillescu, Z. Phys. B 91, 357 (1993) 
[19] M.M. Bredov, B.A. Kotov, N.M. Okuneva, V.S. Oskotskii, and Shak-Budagov, Sov. Phys. Solid State 9, 214 (1967)

[20] S.N. Taraskin and S.R. Elliott, Phys. Rev. B 55, 117 (1997)

[21] J. Dawidowski, F.J. Bermejo, and J.R. Granada, Phys. Rev. B, 58, 706 (1998)

[22] W. Reichardt, MUPHOCOR, a Fortran Program to Determine the Phonon Density of States from Neutron Scattering Experiments, Bericht des Forschungszentrums Karlsruhe, Karlsruhe, Germany (1984)

[23] J. Dawidowski, F.J. Bermejo, R. Fayod, R. Fernández Perea, S.M. Bennington, and A. Criado, Phys. Rev. E 53, $5079(1996)$

[24] J. Wuttke, Phys. Rev. E 62, 6531 (2000)

[25] S.W. Lovesey, Theory of Neutron Scattering from Condensed Matter, Vol. 1, Oxford Science Publishers, Oxford (1984)

[26] J. Kulda, B. Dorner, B. Roessli, H. Sterner, R. Bauer, Th. May, K. Karch, P. Pavone, and D. Strauch, Solid State Commun. 99, 799 (1996)

[27] H. Schober and B. Renker, Phys. Rev. B 59, 3287 (1999)

[28] B. Renker, H. Schober, R. Heid, and P.v. Stein, Solid State Comm. 104, 527 (1997)

[29] M.S. Dresselhaus, et al., Science of Fullerenes and Carbon Nanotubes, Academic Press, San Diego (1996)

[30] A.M. Rao, Z. Ping, W. Kai-An, G.T. Hager, J.M. Holden, Y. Wang, W.-T. Lee, B. Xiang-Xin, P.C. Eklund, D.S. Cornett, M.A. Duncan, and I.J. Amster, Science 259, 955 (1993)

[31] Y. Iwasa, T. Arima, R.M. Fleming, T. Siegrist, O. Zhou, R.C. Haddon, L.J. Rothberg, K.B. Lyons, H.L. Carter, A.F. Hebard, R. Tycko, G. Dabbagh, J.J. Krajewski, G.A. Thomas, and T. Yagi, Science 264, 1570 (1994)

[32] P.W. Stephens, G. Bortel, G. Faigel, M. Tegze, A. Jánossy, S. Pekker, G. Oszlányi, and L. Forró, Nature 370, 636 (1994)

[33] G. Oszlányi et al., Phys. Rev. Lett. 78, 4438 (1997)

[34] H. Schober and B. Renker, Solid State Comm. 104, 609-613 (1997)

[35] L. Pintschovius and S.L. Chaplot, Z. Phys. B. 98, 527 (1995) 